\title{
ON EVALUATING THE INF-SUP CONDITION FOR PLATE BENDING ELEMENTS
}

\author{
ALEXANDER IOSILEVICH, ${ }^{1}$ KLAUS-JÜRGEN BATHE ${ }^{1 *}$ AND FRANCO BREZZI ${ }^{2}$ \\ ${ }^{1}$ Massachusetts Institute of Technology, Department of Mechanical Engineering, Cambridge, MA 02139, U.S.A. \\ ${ }^{2}$ Istituto di Analisi Numerica del Consiglio Nazionale delle Ricerche, 27100 Pavia, Italy
}

\begin{abstract}
This paper addresses the evaluation of the inf-sup condition for Reissner-Mindlin plate bending elements. This fundamental condition for stability and optimality of a mixed finite element scheme is, in general, very difficult to evaluate analytically, considering for example distorted meshes. Therefore, we develop a numerical test methodology. To demonstrate the test methodology and to obtain specific results, we apply it to standard displacement-based elements and elements of the MITC family. Whereas the displacement-based elements fail to satisfy the inf-sup condition, we find that the MITC elements pass our numerical test for uniform meshes and a sequence of distorted meshes. (C) 1997 John Wiley \& Sons, Ltd.
\end{abstract}

Int. J. Numer. Meth. Engng., 40, 3639-3663 (1997)

No. of Figures: $8 . \quad$ No. of Tables: $0 . \quad$ No. of References: 19.

KEY WORDS: Reissner-Mindlin plate elements; inf-sup condition

\section{INTRODUCTION}

Many plate bending finite elements have been proposed since the first application of finite element analysis to plate bending problems. The elements are mostly based on the Kirchhoff and ReissnerMindlin plate theories, and a number of variational formulations have been developed to obtain efficient elements.

The Reissner-Mindlin model offers some advantages in the design of finite elements, namely the theory is applicable to moderately thick to very thin plates, and only the displacement functions and not their derivatives need to satisfy the interelement continuity conditions.

These advantages have spurred much attention to using the Reissner-Mindlin theory as the basis of formulating general plate bending elements. A straightforward way to proceed is to use isoparametric interpolations for the transverse displacement and section rotations. However, this approach leads to well-known difficulties as the plate thickness $t$ is decreased. In analysis of thin plates, the finite element discretization becomes too stiff, which is a numerical phenomenon referred to as shear locking. ${ }^{1,2}$

A number of approaches (and, correspondingly, finite elements) have been proposed in the literature to overcome this purely numerical difficulty; among them are selective/reduced integration schemes, stabilization methods, and various mixed/hybrid formulations.

* Correspondence to: K. J. Bathe, Department of Mechanical Engineering, Massachusetts Institute of Technology, Cambridge, MA 02139, U.S.A.

CCC 0029-5981/97/193639-25\$17.50

Received 23 September 1996

(C) 1997 John Wiley \& Sons, Ltd. 
However, in almost all cases, these elements were solely proposed as efficient because, when used in the solution of certain plate bending problems, accurate results were obtained. A deeper mathematical analysis that ensures stability and convergence properties of the chosen finite element spaces in general analysis conditions has not been performed. Yet, such mathematical analysis is most valuable to firmly prove the reliability of an element.

Among the proposed elements, the MITC element family can be clearly distinguished, because the elements of this family show superior performance in numerical simulations and have a strong mathematical basis. The elements were originally designed as an extension of a four-node mixedinterpolated element using an analogy to analysis of incompressible media. ${ }^{3,4}$ In the construction, the properties of the mixed plate bending elements were deduced from the properties of effective mixed elements for incompressible elasticity, and some error bounds were established and compared with numerical results. ${ }^{5-7}$ For more recent mathematical results on the elements, see the papers by Brezzi et al., ${ }^{8}$ Stenberg and Suri, ${ }^{9}$ and Pitkäranta and Suri. ${ }^{10}$

The two crucial conditions to satisfy for stability and optimal convergence of mixed methods are the ellipticity and inf-sup conditions. ${ }^{1,11}$ If these conditions are satisfied, the finite element discretization is reliable and optimal for the element interpolations used. Thus, it is a great attribute for a mixed finite element formulation to satisfy these conditions. While in many cases it is relatively easy to check whether the ellipticity condition holds, it can be difficult, and indeed almost impossible, to analytically show that the inf-sup condition is satisfied for a given finite element discretization. For this reason, Chapelle and Bathe proposed a numerical inf-sup test for incompressible media analysis. ${ }^{12}$

Our objective in this paper is to propose a numerical scheme for the evaluation of the inf-sup condition for plate bending elements. While we use the test to study whether specifically the MITC elements satisfy the inf-sup condition, the methodology presented is quite general and can also be applied to other discretization schemes. The results given in the paper show that, for uniform discretizations, and a sequence of distorted meshes, the numerical inf-sup test is passed by the MITC elements.

\section{GOVERNING EQUATIONS}

\subsection{Reissner-Mindlin plate model}

Let $\Omega$ be a plane domain with sufficiently smooth (e.g., Lipschitz-continuous) boundary $\partial \Omega$. In the further analysis we will concentrate, without loss of generality, on the case of Dirichlet boundary conditions (clamped plates). The following variational problem governs the model:

$$
\begin{aligned}
& \text { Find } \mathbf{u}=(\boldsymbol{\beta}, w) \in V=B \times W=\left[H_{0}^{1}(\Omega)\right]^{2} \times H_{0}^{1}(\Omega) \text { such that } \\
& \qquad \begin{aligned}
a(\boldsymbol{\beta}, \boldsymbol{\eta})+\frac{\mu k}{t^{2}}(\nabla w-\boldsymbol{\beta}, \nabla \xi-\boldsymbol{\eta})=\frac{1}{t^{3}}(f, \xi) \quad \forall \mathbf{v}=(\boldsymbol{\eta}, \xi) \in V
\end{aligned}
\end{aligned}
$$

where $\boldsymbol{\beta}$ is the vector of rotations $\beta_{1}, \beta_{2} ; w$ is the transverse displacement; and $a(\cdot, \cdot)$ is a symmetric bilinear form defined as

$$
a\left(\boldsymbol{\eta}_{1}, \boldsymbol{\eta}_{2}\right)=\frac{D}{t^{3}} \int_{\Omega}\left[(1-v) \boldsymbol{\varepsilon}\left(\boldsymbol{\eta}_{1}\right): \boldsymbol{\varepsilon}\left(\boldsymbol{\eta}_{2}\right)+v\left(\nabla \cdot \boldsymbol{\eta}_{1}\right)\left(\nabla \cdot \boldsymbol{\eta}_{2}\right)\right] \mathrm{d} \Omega
$$


$D=E t^{3} /\left[12\left(1-v^{2}\right)\right]$ is the flexural rigidity of the plate; $\boldsymbol{\varepsilon}(\cdot)$ is the linear strain operator: $\boldsymbol{\varepsilon}(\boldsymbol{\eta})=$ $\eta_{1,1} \mathbf{e}_{1} \mathbf{e}_{1}+\eta_{1,2} \mathbf{e}_{1} \mathbf{e}_{2}+\eta_{2,1} \mathbf{e}_{2} \mathbf{e}_{1}+\eta_{2,2} \mathbf{e}_{2} \mathbf{e}_{2} ; E, \mu=E /[2(1+v)]$, and $v$ are the Young's modulus, shear modulus, and Poisson's ratio, respectively; $\nabla=\frac{\partial}{\partial x_{1}} \mathbf{e}_{1}+\frac{\partial}{\partial x_{2}} \mathbf{e}_{2} ;(\cdot, \cdot)$ stands for the $L^{2}(\Omega)$ inner product; $f$ is a distributed load acting normal to the plate surface; and $k$ stands for the shear correction factor that accounts for nonuniformity of the shear stress distribution through the plate thickness, usually $k=\frac{5}{6}$.

If we define

$$
\gamma=\frac{\mu k}{t^{2}}(\nabla w-\boldsymbol{\beta})
$$

and $g=f / t^{3}$, we arrive at the variational formulation, equivalent to (1):

Find $\mathbf{u}=(\boldsymbol{\beta}, w) \in V=B \times W=\left[H_{0}^{1}(\Omega)\right]^{2} \times H_{0}^{1}(\Omega)$ and $\boldsymbol{\gamma} \in G$ such that

$$
\begin{aligned}
a(\boldsymbol{\beta}, \boldsymbol{\eta})+(\boldsymbol{\gamma}, \nabla \xi-\boldsymbol{\eta}) & =(g, \xi) \quad \forall \mathbf{v}=(\boldsymbol{\eta}, \xi) \in V \\
\left(\frac{t^{2}}{\mu k} \boldsymbol{\gamma}-(\nabla w-\boldsymbol{\beta}), \boldsymbol{\varsigma}\right) & =0 \quad \forall \boldsymbol{\varsigma} \in G
\end{aligned}
$$

The key point is to identify the appropriate space $G$ for the shear term $\gamma$. Clearly, $\nabla w \in L^{2}(\Omega)$, which implies that for the case of finite plate thickness $t, \gamma \in \nabla W \oplus B \subset L^{2}(\Omega)$. However, as we approach the limit letting $t \rightarrow 0$, the $L^{2}$ regularity of the shear term $\gamma$ is lost, i.e., $\|\gamma\|_{L^{2}}$ is no longer finite. This fact suggests that we should look for a space for shears among the negative Sobolev spaces, and the appropriate space would be the smallest, in which the corresponding norm is finite for all thicknesses, i.e., $\|\gamma\|_{G} \leqslant$ const independent on $t$.

In Appendix I we present a result which manifests that the functional space $\Gamma^{\prime}=H^{-1}(\operatorname{div} ; \Omega)$ satisfies the requirement given above, ${ }^{3}$ and

$$
\begin{aligned}
\Gamma^{\prime}=H^{-1}(\operatorname{div} ; \Omega) & =\left\{\boldsymbol{s}, \boldsymbol{\varsigma} \in\left[H^{-1}(\Omega)\right]^{2}, \nabla \cdot \boldsymbol{\varsigma} \in H^{-1}(\Omega)\right\} \\
\|\boldsymbol{s}\|_{H^{-1}(\operatorname{div} ; \Omega)}^{2} & =\|\boldsymbol{s}\|_{H^{-1}}^{2}+\|\nabla \cdot \boldsymbol{s}\|_{H^{-1}}^{2}
\end{aligned}
$$

As we shall see in Appendix I, $\Gamma^{\prime}$ can also be identified as the dual space of $\Gamma=H_{0}(\operatorname{rot} ; \Omega)$, which is defined as

$$
\begin{gathered}
H_{0}(\operatorname{rot} ; \Omega)=\left\{\chi, \chi \in\left[L^{2}(\Omega)\right]^{2}, \operatorname{rot} \chi \in L^{2}(\Omega), \boldsymbol{\chi} \cdot \tau=0 \text { on } \partial \Omega\right\} \\
\|\boldsymbol{\chi}\|_{H_{0}(\operatorname{rot} ; \Omega)}^{2}=\|\boldsymbol{\chi}\|_{L^{2}}^{2}+\|\operatorname{rot} \boldsymbol{\chi}\|_{L^{2}}^{2}
\end{gathered}
$$

Moreover, we have the following dual norm:

$$
\|\boldsymbol{s}\|_{\Gamma^{\prime}}=\sup _{\chi \in \Gamma} \frac{(\boldsymbol{s}, \boldsymbol{\chi})}{\|\chi\|_{\Gamma}}
$$

If the estimate $\|\boldsymbol{s}\|_{G} \leqslant$ const has to hold independently of $t$, it is reasonable to require it to hold for the limit problem, which is obtained by setting $t=0$ in (3), that is

$$
\begin{aligned}
\text { Find } \mathbf{u}=(\boldsymbol{\beta}, w) \in V & =B \times W=\left[H_{0}^{1}(\Omega)\right]^{2} \times H_{0}^{1}(\Omega) \text { and } \boldsymbol{\gamma} \in \Gamma^{\prime} \text { such that } \\
a(\boldsymbol{\beta}, \boldsymbol{\eta})+(\boldsymbol{\gamma}, \nabla \xi-\boldsymbol{\eta}) & =(g, \xi) \quad \forall \mathbf{v}=(\boldsymbol{\eta}, \xi) \in V \\
(\nabla w-\boldsymbol{\beta}, \boldsymbol{\varsigma}) & =0 \quad \forall \boldsymbol{\varsigma} \in \Gamma^{\prime}
\end{aligned}
$$


Since this problem can clearly serve as the most severe test on the elements' 'addiction' to locking, we will further concentrate on the analysis of equations (7), presuming that, if our finite element discretization provides a good solution for this case, then the elements will not lock under any other circumstances.

Realizing that equations (7) represent, in fact, a particular case of the well-studied saddle-point optimization problem (see e.g., Reference 13), we can focus on the mathematical conditions for existence, uniqueness, stability, and optimality of the solution to (7).

The first, so-called ellipticity condition,

$$
a(\boldsymbol{\eta}, \boldsymbol{\eta}) \geqslant \alpha\|\mathbf{v}\|_{V}^{2}=\alpha\left(\|\boldsymbol{\eta}\|_{H^{1}}^{2}+\|\xi\|_{H^{1}}^{2}\right) \quad \forall \mathbf{v}=(\boldsymbol{\eta}, \xi) \in V_{0}
$$

where $\alpha=$ const $>0$, and

$$
V_{0}=\left\{\mathbf{v}=(\boldsymbol{\eta}, \xi) \in V \mid(\nabla \xi-\boldsymbol{\eta}, \boldsymbol{\varsigma})=0 \quad \forall \boldsymbol{\varsigma} \in \Gamma^{\prime}\right\}
$$

ensures the positive definiteness (after eliminating rigid body modes) of the energy functional in pure bending conditions, and therefore, guarantees existence and uniqueness of $\boldsymbol{\beta}$ and $w$.

Once the ellipticity condition is satisfied, the second, the inf-sup condition,

$$
\inf _{\boldsymbol{\varsigma} \in \Gamma^{\prime}} \sup _{\mathbf{v}=(\boldsymbol{\eta}, \xi) \in V} \frac{(\boldsymbol{\varsigma}, \nabla \xi-\boldsymbol{\eta})}{\|\mathbf{v}\|_{V}\|\boldsymbol{\zeta}\|_{\Gamma^{\prime}}} \geqslant k_{0}>0
$$

where $k_{0}$ is a constant, establishes a criterion for existence, uniqueness, stability and optimality of the solution of equation (7).

\subsection{Finite element approximation. Modified variational problem}

Let us choose two finite element spaces, $W_{h} \subset W$, and $B_{h} \subset B$; then $V_{h}=B_{h} \times W_{h} \subset V$, and we are left with a conforming approximation of the original continuous spaces. The general approach to circumvent the locking behavior is to modify the variational setup (1) by introducing a reduction operator $R_{h}$ so that the problem becomes:

$$
\begin{gathered}
\text { Find } \mathbf{u}_{h}=\left(\boldsymbol{\beta}_{h}, w_{h}\right) \in V_{h}=B_{h} \times W_{h} \text { such that } \\
a\left(\boldsymbol{\beta}_{h}, \mathbf{\eta}_{h}\right)+\frac{\mu k}{t^{2}}\left(R_{h}\left(\nabla w_{h}-\boldsymbol{\beta}_{h}\right), R_{h}\left(\nabla \xi_{h}-\boldsymbol{\eta}_{h}\right)\right)=\left(g, \xi_{h}\right) \quad \forall \mathbf{v}_{h}=\left(\boldsymbol{\eta}_{h}, \xi_{h}\right) \in V_{h}
\end{gathered}
$$

If $R_{h}=I$ (identity operator), we have the standard displacement-based method; if the reduction is based on an inaccurate numerical integration in evaluating the shear energy term, we in essence use the idea of selective or reduced integration. In the following, we will consider the mixed interpolation approach, in which the shear terms are first approximated independently, and then eliminated from the system.

Defining the discrete approximation for the shear term as

$$
\gamma_{h}=\frac{\mu k}{t^{2}} R_{h}\left(\nabla w_{h}-\boldsymbol{\beta}_{h}\right)
$$


we have an equivalent discrete variational problem:

$$
\begin{aligned}
& \text { Find } \mathbf{u}_{h}=\left(\boldsymbol{\beta}_{h}, w_{h}\right) \in V_{h}=B_{h} \times W_{h} \text { and } \gamma_{h} \in G_{h}=R_{h}\left(V_{h}\right) \text { such that } \\
& \begin{aligned}
a\left(\boldsymbol{\beta}_{h}, \boldsymbol{\eta}_{h}\right)+\left(\gamma_{h}, R_{h}\left(\nabla \xi_{h}-\boldsymbol{\eta}_{h}\right)\right) & =\left(g, \xi_{h}\right) \quad \forall \mathbf{v}_{h}=\left(\boldsymbol{\eta}_{h}, \xi_{h}\right) \in V_{h}
\end{aligned} \\
& \left(\frac{t^{2}}{\mu k} \gamma_{h}-R_{h}\left(\nabla w_{h}-\boldsymbol{\beta}_{h}\right), \boldsymbol{\varsigma}_{h}\right)=0 \quad \forall \boldsymbol{\varsigma}_{h} \in G_{h}
\end{aligned}
$$

Following the same procedure as in the previous section, we can define the discrete limit problem:

Find $\mathbf{u}_{h}=\left(\boldsymbol{\beta}_{h}, w_{h}\right) \in V_{h}=B_{h} \times W_{h}$ and $\gamma_{h} \in G_{h}$ such that

$$
\begin{aligned}
a\left(\boldsymbol{\beta}_{h}, \boldsymbol{\eta}_{h}\right)+\left(\gamma_{h}, R_{h}\left(\nabla \xi_{h}-\boldsymbol{\eta}_{h}\right)\right) & =\left(g, \xi_{h}\right) \quad \forall \mathbf{v}_{h}=\left(\boldsymbol{\eta}_{h}, \xi_{h}\right) \in V_{h} \\
\left(R_{h}\left(\nabla w_{h}-\boldsymbol{\beta}_{h}\right), \boldsymbol{\zeta}_{h}\right) & =0 \quad \forall \boldsymbol{\varsigma}_{h} \in G_{h}
\end{aligned}
$$

The corresponding conditions for existence, uniqueness, stability and optimality of the solution to (13) are given by

$$
a\left(\boldsymbol{\eta}_{h}, \boldsymbol{\eta}_{h}\right) \geqslant \alpha\left\|\mathbf{v}_{h}\right\|_{V}^{2}=\alpha\left(\left\|\boldsymbol{\eta}_{h}\right\|_{H^{1}}^{2}+\left\|\xi_{h}\right\|_{H^{1}}^{2}\right) \quad \forall \mathbf{v}_{h}=\left(\boldsymbol{\eta}_{h}, \xi_{h}\right) \in V_{0 h}
$$

where

$$
V_{0 h}=\left\{\mathbf{v}_{h}=\left(\boldsymbol{\eta}_{h}, \xi_{h}\right) \in V_{h} \mid\left(R_{h}\left(\nabla \xi_{h}-\boldsymbol{\eta}_{h}\right), \boldsymbol{\varsigma}_{h}\right)=0 \forall \boldsymbol{s}_{h} \in G_{h}\right\}
$$

and

$$
\inf _{\boldsymbol{\varsigma}_{h} \in G_{h}} \sup _{\mathbf{v}_{h}=\left(\mathbf{\eta}_{h}, \xi_{h}\right) \in V_{h}} \frac{\left(\boldsymbol{s}_{h}, R_{h}\left(\nabla \xi_{h}-\boldsymbol{\eta}_{h}\right)\right)}{\left\|\mathbf{v}_{h}\right\|_{V}\left\|\boldsymbol{s}_{h}\right\|_{\Gamma^{\prime}}} \geqslant k_{0}>0
$$

Notice that (14) will follow easily if we require that

(i) $\exists c_{1}>0$ such that $\left\|R_{h} \mathbf{v}_{h}\right\|_{L^{2}} \leqslant c_{1}\left\|\mathbf{v}_{h}\right\|_{L^{2}} \forall \mathbf{v}_{h} \in V_{h}$; and

(ii) $\exists c_{2}>0$ such that $\left\|R_{h} \nabla \xi_{h}\right\|_{L^{2}} \geqslant c_{2}\left\|\nabla \xi_{h}\right\|_{L^{2}} \forall \xi_{h} \in W_{h}$

Condition (i) just enforces continuity of the reduction operator $R_{h}$, and is quite natural due to the physics of the problem. Actually, in order to have optimal results, we shall also need that $R_{h}$ is 'a good approximation of the identity'. For instance, we might ask that

$$
\left\|R_{h} \mathbf{v}-\mathbf{v}\right\|_{L^{2}} \leqslant c h^{k}
$$

for every smooth $\mathbf{v}$ in $V$ ( $k$ being the order of the interpolation space $\left.V_{h}\right)$ and

$$
\left(R_{h} \mathbf{v}_{h}-\mathbf{v}_{h}, \boldsymbol{\varsigma}\right) \leqslant c\left\|\mathbf{v}_{h}\right\|_{V} h^{k}
$$

for every $\mathbf{v}_{h} \in V_{h}$ and for every smooth $\varsigma$ in $H_{0}(\operatorname{rot} ; \Omega)$, (see (5)).

On the other hand, (ii) says that, in a certain sense, we do not loose too much information on transverse displacements by considering $R_{h} \nabla w_{h}$ instead of $\nabla w_{h}$. Note that if we had (in contrast with (ii)) $R_{h} \nabla \widetilde{\xi}_{h}=0$ for some $\widetilde{\xi}_{h} \in W_{h} \backslash\{0\}$, then $\widetilde{\xi}_{h}$ would be a parasitic mode, displaying zero energy in (10). 
If (i) and (ii) hold, then (14) will also hold, and we have only to check (15). If indeed (15) is satisfied, then we have

$$
\left\|\boldsymbol{\beta}-\boldsymbol{\beta}_{h}\right\|_{H^{1}}+\left\|w-w_{h}\right\|_{H^{1}}+\left\|\boldsymbol{\gamma}-\gamma_{h}\right\|_{\Gamma^{\prime}} \leqslant c h^{k}
$$

uniformly in $t$, provided the solution $(\boldsymbol{\beta}, w, \gamma)$ of (3) is smooth and (16)-(17) hold as well.

Remark 1. In a general case, the solution of (3) will not be smooth enough (uniformly in $t$ ) to give (18), as pointed out in Reference 14, due to boundary layer effects. However, on one hand (18) still provides reliable information on the potential accuracy of the method; on the other hand, in many cases one can have interior estimates of type (18) even in the presence of boundary layers, as pointed out by Gastaldi. ${ }^{15}$

\section{MITC PLATE BENDING ELEMENTS}

In this section we present the MITCn family of plate bending elements, following the original papers. ${ }^{4,5,7}$

\subsection{Design principles}

For the choice of operator $R_{h}$, we still need (i), (ii), (16) and (17) to hold true. As we shall see, (i), (16) and (17) will come out naturally from our other choices, while in order to enforce (ii) we take a stronger condition

$$
\left(R_{h}-I\right) \nabla \xi_{h}=\mathbf{0} \quad \forall \xi_{h} \in W_{h}
$$

This assumption (which clearly implies (i)) is rather strong but, as we shall see, it has the merit of simplifying greatly the design procedure.

In the limit case $t=0$ the continuous solution $(\boldsymbol{\beta}, w, \gamma)$ will satisfy the Kirchhoff constraint $\nabla w=\boldsymbol{\beta}$, and the discrete solution will satisfy

$$
R_{h}\left(\nabla w_{h}-\boldsymbol{\beta}_{h}\right)=\mathbf{0}
$$

The least we can ask is that for every smooth pair $\nabla w=\boldsymbol{\beta}$ there exists at least a discrete pair $\left(\boldsymbol{\beta}_{h}, w_{h}\right)$ satisfying (20) and (optimally) close to $(\boldsymbol{\beta}, w)$. In order to have (20) we need (using (19))

$$
\nabla w_{h}=R_{h} \boldsymbol{\beta}_{h}
$$

and recalling that for any scalar function $\varphi$,

$$
\operatorname{rot} \nabla \varphi=\nabla \times \nabla \varphi=0
$$

we can equivalently rewrite condition (21) as

$$
\operatorname{rot} R_{h} \boldsymbol{\beta}_{h}=0
$$

For the time being, we consider a slightly different constraint, namely

$$
\left\{\left(\operatorname{rot} \boldsymbol{\beta}_{h}, q\right)=0 \forall q \in Q_{h} \subset Q=L^{2}(\Omega)\right\} \quad \text { or } \quad P_{h} \operatorname{rot} \boldsymbol{\beta}_{h}=0
$$


where $P_{h}$ stands for the projection operator defined over a discrete space $Q_{h} \in L^{2}(\Omega)$. Noting that for a scalar function $\varphi$ and vector function $\mathbf{v}$, we have

$$
\operatorname{rot} \varphi=(\nabla \varphi)^{\perp}, \quad \operatorname{rot} \mathbf{v}=\nabla \cdot \mathbf{v}^{\perp}
$$

where ' $\perp$ ' is the ninety degree clockwise rotation operator, we can consider an auxiliary variational problem, similar to the one encountered in the analysis of incompressible media:

$$
\begin{aligned}
& \text { Find } \boldsymbol{\beta}_{h} \in B_{h} \text { and } p_{h} \in Q_{h} \subset L^{2}(\Omega), \text { such that } \\
& a^{S}\left(\boldsymbol{\beta}_{h}, \boldsymbol{\eta}_{h}\right)-\left(\operatorname{rot} \boldsymbol{\eta}_{h}, p_{h}\right)=a^{S}\left(\boldsymbol{\beta}, \boldsymbol{\eta}_{h}\right) \quad \forall \boldsymbol{\eta}_{h} \in B_{h} \\
& \left(\operatorname{rot} \boldsymbol{\beta}_{h}, q_{h}\right)=0 \quad \forall q_{h} \in Q_{h}
\end{aligned}
$$

where $a^{S}\left(\boldsymbol{\eta}_{1}, \boldsymbol{\eta}_{2}\right)=\int_{\Omega} \nabla \boldsymbol{\eta}_{1}: \nabla \boldsymbol{\eta}_{2} \mathrm{~d} \Omega$.

To have a unique solution for $\boldsymbol{\beta}_{h}$ and $p_{h}$ and optimal accuracy for $\left\|\boldsymbol{\beta}-\boldsymbol{\beta}_{h}\right\|$, our choice of the discrete spaces $\left(B_{h}, Q_{h}\right)$ has to satisfy the inf-sup condition

$$
\inf _{q_{h} \in Q_{h}} \sup _{\boldsymbol{\eta}_{h} \in B_{h}} \frac{\left(\operatorname{rot} \boldsymbol{\eta}_{h}, q_{h}\right)}{\left\|q_{h}\right\|_{Q}\left\|\boldsymbol{\eta}_{h}\right\|_{B}} \geqslant k_{0}>0
$$

with the constant $k_{0}$ independent of the typical mesh size $h$. Using relations (25), we conclude that to have a unique, stable and optimally convergent solution in (26), our discrete spaces must satisfy the inf-sup condition for the case of incompressibility

$$
\inf _{q_{h} \in Q_{h}} \sup _{\boldsymbol{\eta}_{h} \in B_{h}} \frac{\left(\nabla \cdot \boldsymbol{\eta}_{h}, q_{h}\right)}{\left\|q_{h}\right\|_{Q}\left\|\boldsymbol{\eta}_{h}\right\|_{B}} \geqslant k_{0}>0
$$

There exist quite a few pairs $\left(B_{h}, Q_{h}\right)$ described in the literature, that are known to satisfy the inf-sup condition (28) (see Reference 13). Therefore, taking such a pair, we have that, for every smooth pair $\boldsymbol{\beta}=\nabla w$ we can construct a $\boldsymbol{\beta}_{h} \in B_{h}$, such that $\boldsymbol{\beta}_{h} \rightarrow \boldsymbol{\beta}$ with an optimal rate, and $P_{h}$ rot $\boldsymbol{\eta}_{h}$ (that is (24)). In order to have (23), we must now require

$$
P_{h} \operatorname{rot} \boldsymbol{\eta}_{h}=\operatorname{rot} R_{h} \boldsymbol{\eta}_{h} \in Q_{h}
$$

Finally, (23) will imply the existence of a $w_{h} \in W_{h}$ satisfying (21) if we require that $W_{h}$ satisfy

$$
\nabla W_{h}=\left\{\zeta_{h} \in \Gamma_{h}, \operatorname{rot} \zeta_{h}=0\right\}
$$

Notice that (30) will imply (19) as soon as $R_{h}=I$ when restricted to $\Gamma_{h}$.

Thus, we can summarize the design process as follows:

1. Start with a pair of functional spaces $\left(B_{h}, Q_{h}\right)$ that satisfies the inf-sup condition (28). $B_{h}$ would be the space of section rotations, while $Q_{h}$ is an auxiliary space, which never appears in actual calculations. 
2. Find another space $\Gamma_{h} \subset \Gamma$ and a reduction operator $R_{h}$, such that the following diagram commutes:

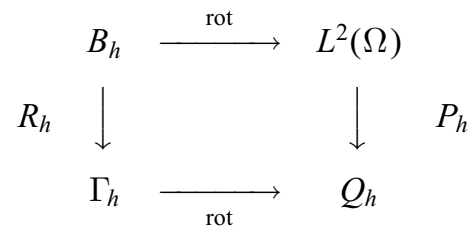

This condition is referred to as the commuting diagram property, and is equivalent to (29). 3. Choose $W_{h}$ to satisfy condition (30) and yield the proper order of accuracy.

Remark 2. As was pointed out above, the functional space $Q_{h}$ does not have any physical meaning, and serves just as a link between the Stokes system and the problem under consideration. Since we are barely interested in the solution for $p_{h}$ in (26), the inf-sup condition (28) which ensures solvability, stability, and optimal convergence of both $\boldsymbol{\beta}_{h}$ and $p_{h}$ seems to be too strong, and we should not discard the pairs $\left(B_{h}, Q_{h}\right)$ which are known to work for velocities, but fail to produce stable results for pressures in the Stokes problem. A weaker condition to guarantee the solvability would be the stability inequality

$$
\sup _{\boldsymbol{\eta}_{h} \in B_{h}} \frac{\left(\nabla \cdot \boldsymbol{\eta}_{h}, q_{h}\right)}{\left\|\boldsymbol{\eta}_{h}\right\|_{B}} \geqslant c(h)\left\|q_{h}\right\|_{Q} \quad \forall q_{h} \in Q_{h}, \quad q_{h} \neq 0
$$

It can be demonstrated (see Reference 13 for the proof) that elements, which are designed following the procedure stated above, satisfy a weak form of the inf-sup condition (15),

$$
\inf _{\boldsymbol{\varsigma}_{h} \in \Gamma_{h}} \sup _{\mathbf{v}_{h}=\left(\boldsymbol{\eta}_{h}, \xi_{h}\right) \in V_{h}} \frac{\left(\boldsymbol{\zeta}_{h}, R_{h}\left(\nabla \xi_{h}-\boldsymbol{\eta}_{h}\right)\right)}{\left\|\mathbf{v}_{h}\right\|_{V}\left\|\boldsymbol{S}_{h}\right\|_{\Gamma_{h}^{\prime}}^{\prime}} \geqslant \beta>0
$$

with $\beta$ not dependent on the mesh size and plate thickness, and where the $\Gamma_{h}^{\prime}$-norm is defined as

$$
\left\|\boldsymbol{s}_{h}\right\|_{\Gamma_{h}^{\prime}}=\sup _{\chi_{h} \in \Gamma_{h}} \frac{\left(\boldsymbol{s}_{h}, \chi_{h}\right)}{\left\|\boldsymbol{\chi}_{h}\right\|_{\Gamma}}
$$

We will refer to equation (33) as the inf-sup condition in the $\Gamma_{h}^{\prime}$-norm.

The main implication of this result is that in case of uniform meshes, the following error bounds hold: ${ }^{13}$

$$
\left\|\boldsymbol{\beta}-\boldsymbol{\beta}_{h}\right\|_{H^{1}}+\left\|w-w_{h}\right\|_{H^{1}}+\left\|\gamma-\gamma_{h}\right\|_{\Gamma_{h}^{\prime}} \leqslant c h^{k}
$$

where $k$ is the order of the interpolation space $V_{h}$.

Clearly, to satisfy the actual inf-sup condition (15), and make the bounds (18) applicable, we should have that the following condition holds:

$$
\left\|\boldsymbol{s}_{h}\right\|_{\Gamma_{h}^{\prime}} \geqslant \rho\left\|\boldsymbol{s}_{h}\right\|_{\Gamma^{\prime}} \quad \forall \boldsymbol{s}_{h} \in \Gamma_{h}
$$

with a constant $\rho$ independent of the plate thickness $t$ and characteristic mesh size $h$. 
So far no analytical results exist regarding the norm equivalence (36) and regarding the inf-sup condition (15). Such results would be very hard, if not impossible, to obtain for general distorted meshes, and we therefore resort to a numerical treatment.

\subsection{The elements}

In this section we briefly describe the displacement-based approach to the plate bending problem, as well as the MITCn plate bending elements, taking the MITC4 element as a special case, and the MITC9 element as a typical representative of the family.

We define all spaces for a reference $2 \times 2$ square element $K=(-1 ; 1)^{2}$, and these derivations can be directly extended to any general case through covariant transformations (provided the Jacobian matrix of the transformation is not singular) ${ }^{1}$.

3.2.1. Displacement-based finite elements. The simplicity of these elements made them popular among practitioners although the produced results often suffer from locking. The space for shears is obtained as (taking $R_{h}=I$ )

$$
\Gamma_{h}=\nabla W_{h} \oplus B_{h}
$$

which implies

$$
V_{0}=\left\{\mathbf{v}_{h}=\left(\boldsymbol{\eta}_{h}, \xi_{h}\right) \in V_{h} \mid \nabla \xi_{h}=\boldsymbol{\eta}_{h}\right\}
$$

This condition severely restricts the number of functions which satisfy the Kirchhoff constraint to a small subspace of the original space for displacements $V_{h}$, barely leaving a hope to have good convergence properties for all plate thicknesses $t$, especially for lower order elements.

3.2.2. The MITC4 element. The element was introduced by Dvorkin and Bathe ${ }^{16}$ as a general shell element. Results regarding the numerical performance, convergence studies, and an error analysis can be found in References 3 and 17.

For the mixed-interpolated four-node element we use

$$
\begin{aligned}
W_{h} & =\left\{\xi_{h} \in H_{0}^{1}(\Omega),\left.\xi_{h}\right|_{K} \in Q_{1}(K)\right\} \\
B_{h} & =\left\{\boldsymbol{\eta}_{h} \in\left[H_{0}^{1}(\Omega)\right]^{2},\left.\quad \boldsymbol{\eta}_{h}\right|_{K} \in\left[Q_{1}(K)\right]^{2}\right\} \\
Q_{h} & =\left\{q_{h}:\left.q_{h}\right|_{K} \in \mathbb{R}\right\} \\
\Gamma_{h} & =\left\{\boldsymbol{s}_{h} \in H_{0}(\operatorname{rot} ; \Omega),\left.\boldsymbol{\varsigma}_{h}\right|_{K} \in R T(K)\right\} \\
R T & =\{\operatorname{span}\{1, s\}, \operatorname{span}\{1, r\}\}
\end{aligned}
$$

Although the $Q_{1}-P_{0}$ pair satisfies the stability inequality (32) with $c(h)=O(h),{ }^{18}$ it fails to satisfy the inf-sup condition for the Stokes problem (28), so that the analytical results of Section 3.1 are not applicable to the MITC4 case. The existing theoretical error bound for the case of uniform meshes is

$$
\left\|\boldsymbol{\beta}-\boldsymbol{\beta}_{h}\right\|_{H^{1}}+\left\|w-w_{h}\right\|_{H^{1}}+t\left\|\boldsymbol{\gamma}-\gamma_{h}\right\|_{L^{2}} \leqslant c h
$$

and depends on the plate thickness $t$.

3.2.3. MITC9 element. For the mixed-interpolated nine-node element we use: ${ }^{5}$

$$
W_{h}=\left\{\xi_{h} \in H_{0}^{1}(\Omega),\left.\quad \xi_{h}\right|_{K} \in Q_{2}^{\prime}(K)\right\}
$$




$$
\begin{aligned}
B_{h} & =\left\{\boldsymbol{\eta}_{h} \in\left[H_{0}^{1}(\Omega)\right]^{2},\left.\boldsymbol{\eta}_{h}\right|_{K} \in\left[Q_{2}(K)\right]^{2}\right\} \\
Q_{h} & =\left\{q_{h}:\left.q_{h}\right|_{K} \in P_{1}(K)\right\} \\
\Gamma_{h} & =\left\{\boldsymbol{s}_{h} \in H_{0}(\operatorname{rot} ; \Omega),\left.\boldsymbol{s}_{h}\right|_{K} \in B D F M(K)\right\} \\
B D F M & =\left\{\operatorname{span}\left\{1, r, s, r s, s^{2}\right\}, \operatorname{span}\left\{1, r, s, r s, r^{2}\right\}\right\}
\end{aligned}
$$

where $Q_{2}^{\prime}$ is the second order Serendipity space. ${ }^{1}$

The pair $\left(B_{h}, Q_{h}\right)$ is known to satisfy the inf-sup condition (28); indeed, we can check (see the details in Reference 5) that all other requirements of the design scheme (i.e., the commuting diagram property (31) and condition (30)) are satisfied with this choice of functional spaces.

Therefore, the results of Section 3.1 are applicable in this case, and we can conclude that the element satisfies the inf-sup condition in the $\Gamma_{h}^{\prime}$-norm at least for uniform, non-distorted meshes. The theoretically predicted error bound is ${ }^{13}$

$$
\left\|\boldsymbol{\beta}-\boldsymbol{\beta}_{h}\right\|_{H^{1}}+\left\|w-w_{h}\right\|_{H^{1}}+\left\|\gamma-\gamma_{h}\right\|_{\Gamma_{h}^{\prime}} \leqslant c h^{2}
$$

3.2.4. Other elements. The MITCn family of plate bending elements also includes a higher order 16-node quadrilateral element MITC16, and two triangular elements, the MITC7 and MITC12 elements. ${ }^{1}$ All these elements have the same properties as the MITC9 element, namely, they satisfy the ellipticity condition (14) (and hence have no spurious zero eigenvalues), they satisfy the infsup condition in the $\Gamma_{h}^{\prime}$-norm (for uniform meshes) and hence conform to the error bounds (35).

For the summary of elements and numerical results see References 1, 6 and 7.

\section{TEST METHODOLOGY}

In this section we develop a test methodology which allows to check numerically whether the inf-sup condition holds for a particular discretization. In our developments we follow the idea of the numerical inf-sup test proposed by Chapelle and Bathe, ${ }^{1,12}$ and apply the test to the MITC elements and to the displacement-based elements.

As we have demonstrated in the previous section, in order to prove that the inf-sup condition (15) is satisfied for a given finite element discretization, it is sufficient to show that:

(a) The inf-sup condition holds with respect to the $\Gamma_{h}^{\prime}$-norm for the shear term, as given by (33); and

(b) The norm equivalence stated in (36) holds with a constant $\rho$ independent of the plate thickness $t$ and mesh size $h$.

Correspondingly, we propose two numerical tests, which allow to check whether (a) and (b) are satisfied for a given mesh.

\subsection{Inf-sup test in the $\Gamma_{h}^{\prime}$-norm}

We start by establishing a matrix form of the inf-sup condition in the $\Gamma_{h}^{\prime}$-norm. We take the following norm for the space for rotations and transverse displacement $\left\|\mathbf{v}_{h}\right\|_{V}=\left\|\mathbf{v}_{h}\right\|_{H^{1}}$. Restricting our analysis to the case of bounded domains, we have by the Poincaré-Friedrichs inequality: ${ }^{1}$

$$
\left\|\mathbf{v}_{h}\right\|_{V}^{2} \simeq\left|\mathbf{v}_{h}\right|_{H^{1}}^{2}=\left|\boldsymbol{\eta}_{h}\right|_{H^{1}}^{2}+\left|\xi_{h}\right|_{H^{1}}^{2}=L^{2} \sum_{i, j=1}^{2}\left\|\frac{\partial \eta_{h, i}}{\partial x_{j}}\right\|_{L^{2}}^{2}+\sum_{i=1}^{2}\left\|\frac{\partial \xi_{h}}{\partial x_{i}}\right\|_{L^{2}}^{2}=\mathbf{V}^{\mathrm{T}} \mathbf{S V}
$$


where $L$ is a characteristic dimension of the plate (i.e., plate width or length), $\mathbf{V} \in \mathbb{R}^{n}$ is a vector of nodal point displacements, and $\mathbf{S} \in \mathbb{R}^{n} \times \mathbb{R}^{n}$ is a positive definite norm matrix.

Shear terms are calculated from nodal point displacements $\mathbf{U}$ using a shear nodal basis $\mathbf{B}_{\gamma}$ : $\mathbb{R}^{n} \rightarrow \Gamma_{h}$ as $\boldsymbol{\varsigma}_{h}=\mathbf{B}_{\gamma} \mathbf{U}$. Let us define the $L^{2}$-inner product $\mathbf{G}=\left(\mathbf{B}_{\gamma}^{\mathrm{T}}, \mathbf{B}_{\gamma}\right), \mathbf{G} \in \mathbb{R}^{n} \times \mathbb{R}^{n}$; then, the numerator of (33) can be rewritten as $\left(\boldsymbol{\varsigma}_{h}, R_{h}\left(\nabla \xi_{h}-\boldsymbol{\eta}_{h}\right)\right)=\left(\mathbf{U}^{\mathrm{T}} \mathbf{B}_{\gamma}^{\mathrm{T}}, \mathbf{B}_{\gamma} \mathbf{V}\right)=\mathbf{U}^{\mathrm{T}} \mathbf{G V}$, where $\mathbf{G}$ is a positive semidefinite $n \times n$ matrix. To avoid corner solutions (i.e., the inf-sup value being either zero or infinity), we impose the following restriction: $\mathbf{V}, \mathbf{U} \in \mathscr{D}=\mathbb{R}^{n} \backslash \operatorname{ker}(\mathbf{G})$, where $\operatorname{ker}(\mathbf{G})$ stands for the null space of $\mathbf{G}$.

To calculate $\left\|\boldsymbol{S}_{h}\right\|_{\Gamma_{h}^{\prime}}$, we will use the definition (34). Taking $\left\|\boldsymbol{\chi}_{h}\right\|_{\Gamma}=\left\|\mathbf{B}_{\gamma} \mathbf{W}\right\|_{\Gamma}$ as

$$
\left\|\boldsymbol{\chi}_{h}\right\|_{\Gamma}^{2}=\left\|\boldsymbol{\chi}_{h}\right\|_{H_{0}(\mathrm{rot} ; \Omega)}^{2}=\left\|\chi_{h}\right\|_{L^{2}}^{2}+L^{2}\left\|\operatorname{rot} \chi_{h}\right\|_{L^{2}}^{2}=\mathbf{W}^{\mathrm{T}}(\mathbf{G}+\mathbf{Q}) \mathbf{W}=\mathbf{W}^{\mathrm{T}} \mathbf{D} \mathbf{W}
$$

where $\mathbf{Q}$ is a symmetric positive-semidefinite matrix, we can rewrite (34) as

$$
\left\|\boldsymbol{s}_{h}\right\|_{\Gamma_{h}^{\prime}}=\sup _{\mathbf{W} \in \mathscr{D}} \frac{\mathbf{U}^{\mathrm{T}} \mathbf{G W}}{\sqrt{\mathbf{W}^{\mathrm{T}} \mathbf{D W}}}
$$

Of course, the condition $\mathbf{W} \in \mathscr{D}$ is sufficient to guarantee that the seminorm in the denominator can be used as a norm. Summarizing, the inequality (33) can be written in matrix form as

$$
\inf _{\mathbf{U} \in \mathscr{D}} \sup _{\mathbf{V} \in \mathscr{D}} \frac{\mathbf{U}^{\mathrm{T}} \mathbf{G V}}{\sup _{\mathbf{W} \in \mathscr{D}} \frac{\mathbf{U}^{\mathrm{T}} \mathbf{G W}}{\sqrt{\mathbf{W}^{\mathrm{T}} \mathbf{D W}}} \sqrt{\mathbf{V}^{\mathrm{T}} \mathbf{S V}}} \geqslant \beta
$$

We show in Appendix II that the following bound can be derived for the inf-sup value $\beta$ :

$$
\beta \geqslant \beta^{*}=\sqrt{\delta_{\min } / \sigma_{\max }}
$$

where $\delta_{\min }$ stands for the smallest nonzero eigenvalue of $\mathbf{D}$, and $\sigma_{\max }$ is the largest eigenvalue of $\mathbf{S}$.

To analyse the behaviour of an element, we calculate $\beta^{*}$ for a sequence of meshes obtained by successive refinements and plot $\lg \left(\beta^{*}\right)$ vs. $\lg (1 / N)$, where $N$ stands for the square root of the total number of elements. If we see that the value $\beta^{*}$ converges to zero, the element fails the inf-sup test, and thus does not satisfy the inf-sup condition (15). Conversely, if $\beta^{*}$ stabilizes away from zero, we conclude that the test is passed.

\subsection{From $\Gamma_{h}^{\prime}$ to $\Gamma^{\prime}$}

We refine the finite element space with a characteristic mesh size $h$, simply by transforming each element $j, j=1, \ldots, N^{2}$ to the reference element $K$ through a covariant transformation, then subdividing $K$ into four 'subelements' $K_{i}, i=1 \ldots 4$, and transforming the resulting functional space back to $j$ (Figure 1 demonstrates this procedure for a four-node element). We call the resulting space for the shear term $\Gamma_{h / 2}$. The next refinement would give us $\Gamma_{h / 4}$ and so on. Clearly, we have that $\Gamma_{h} \subseteq \Gamma_{h / 2} \subseteq \Gamma_{h / 4} \subseteq \cdots \subseteq \Gamma_{h / n} \subset \Gamma$, and increasing the refinement, we can approach the continuous space $\Gamma$ as closely as we want by our finite element discretization. Moreover, for $\boldsymbol{\varsigma}_{h} \in \Gamma_{h}$, we have

$$
\left\|\boldsymbol{S}_{h}\right\|_{\Gamma_{h / n}^{\prime}}=\sup _{\chi_{h} \in \Gamma_{h / n}} \frac{\left(\boldsymbol{s}_{h}, \chi_{h}\right)}{\left\|\chi_{h}\right\|_{\Gamma_{h / n}}} \underset{n \rightarrow \infty}{\longrightarrow}\left\|\boldsymbol{s}_{h}\right\|_{\Gamma^{\prime}}
$$




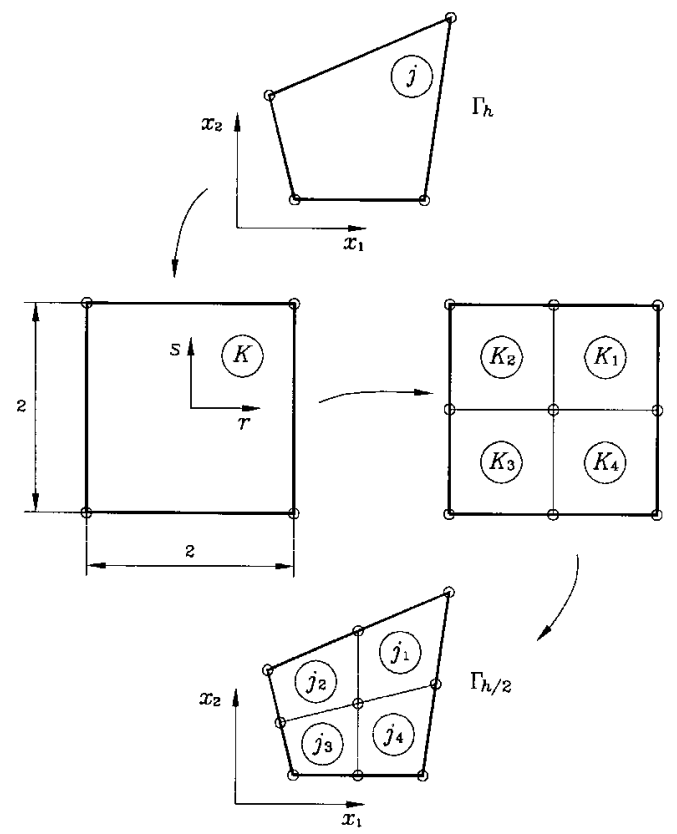

Figure 1. $\Gamma_{h} \rightarrow \Gamma_{h / 2}$ transformation for a reference four-node element

so that, if we consider

$$
\theta_{n}(h)=\inf _{\boldsymbol{S}_{h} \in \Gamma_{h}} \frac{\left\|\boldsymbol{S}_{h}\right\|_{\Gamma_{h}^{\prime}}}{\left\|\boldsymbol{S}_{h}\right\|_{\Gamma_{h / n}^{\prime}}}
$$

we can find an estimate (for large $n$ ) for the constant

$$
\theta_{\infty}(h)=\inf _{\boldsymbol{S}_{h} \in \Gamma_{h}} \frac{\left\|\boldsymbol{S}_{h}\right\|_{\Gamma_{h}^{\prime}}}{\left\|\boldsymbol{S}_{\boldsymbol{h}}\right\|_{\Gamma^{\prime}}}
$$

If the constant $\theta_{\infty}(h)$ does not converge to zero as $h$ tends to zero, that is if

$$
\theta_{\infty}(h) \geqslant \bar{\theta}>0
$$

for some $\bar{\theta}$ independent of $h$, we can conclude that the $\Gamma_{h}^{\prime}$ and the $\Gamma^{\prime}$ norms, for the finite element spaces under analysis, are equivalent (with constants independent of $h$ ), so that the two inf-sup conditions, (15) and (33) are in fact equivalent.

Remark 3. In some cases, it is possible to prove analytically that there exists a constant $c$, independent of $h$, such that ${ }^{19}$

$$
\theta_{2}(h) \leqslant c \theta_{\infty}(h)
$$


so that, in practice, it is sufficient to check whether $\theta_{2}(h)$ is bounded from below by a positive constant independent of $h$ :

$$
\theta_{2}(h) \geqslant c_{1}>0
$$

Clearly, (44) then follows from (45) and (46) with $\bar{\theta}=c_{1} / c$.

Hence, in general, the essence of our test is to evaluate a lower bound for $\theta_{\infty}(h)$ as $h \rightarrow 0$, and we require this bound not to converge to zero.

To compute $\theta_{n}(h)$ we first rewrite it, according to the definitions (34) and (41) as

$$
\theta_{n}(h)=\inf _{\boldsymbol{\varsigma}_{h} \in \Gamma_{h}} \frac{\sup _{\chi_{h} \in \Gamma_{h}} \frac{\left(\boldsymbol{\varsigma}_{h}, \chi_{h}\right)}{\left\|\chi_{h}\right\|_{\Gamma_{h}}}}{\sup _{\mathbf{\kappa}_{h} \in \Gamma_{h / n}} \frac{\left(\boldsymbol{\varsigma}_{h}, \mathbf{\kappa}_{h}\right)}{\left\|\boldsymbol{\kappa}_{h}\right\|_{\Gamma_{h / n}}}}
$$

Then, in order to rewrite (47) in matrix form, we define

$$
\begin{aligned}
\boldsymbol{s}_{h} & =\overline{\mathbf{B}}_{\gamma} \mathbf{U}, \quad \chi_{h}=\overline{\mathbf{B}}_{\gamma} \overline{\mathbf{V}}, \quad \overline{\mathbf{B}}_{\gamma}: \mathbb{R}^{\bar{m}} \rightarrow \Gamma_{h}, \quad \mathbf{U}, \overline{\mathbf{V}} \in \mathbb{R}^{\bar{m}} \\
\left\|\chi_{h}\right\|_{\Gamma_{h}} & =\sqrt{\overline{\mathbf{V}}^{\mathrm{T}} \overline{\mathbf{D}} \overline{\mathbf{V}}}, \quad \overline{\mathbf{G}}=\left(\overline{\mathbf{B}}_{\gamma}^{\mathrm{T}}, \overline{\mathbf{B}}_{\gamma}\right), \quad \overline{\mathbf{D}}, \overline{\mathbf{G}} \in \mathbb{R}^{\bar{m}} \times \mathbb{R}^{\bar{m}} \\
\mathbf{\kappa}_{h} & =\mathbf{B}_{\gamma} \mathbf{V}, \quad \mathbf{B}_{\gamma}: \mathbb{R}^{m} \rightarrow \Gamma_{h / n}, \quad \mathbf{V} \in \mathbb{R}^{m}, \quad\left\|\mathbf{\kappa}_{h}\right\|_{\Gamma_{h / n}}=\sqrt{\mathbf{V}^{\mathrm{T}} \mathbf{D V}} \\
\mathbf{D} & \in \mathbb{R}^{m} \times \mathbb{R}^{m}, \quad \mathbf{G}=\left(\mathbf{B}_{\gamma}^{\mathrm{T}}, \overline{\mathbf{B}}_{\gamma}\right), \quad \mathbf{G} \in \mathbb{R}^{m} \times \mathbb{R}^{\bar{m}} \\
\overline{\mathscr{D}} & =\mathbb{R}^{\bar{m}} \backslash \operatorname{ker}(\overline{\mathbf{G}}), \quad \mathscr{D}=\mathbb{R}^{m} \backslash \operatorname{ker}(\mathbf{D})
\end{aligned}
$$

where $\bar{m}$ and $m$ are dimensions of the nodal point displacement vectors corresponding to the spaces $\Gamma_{h}$ and $\Gamma_{h / n}$, respectively. Substituting these expressions into equation (47), we obtain

$$
\theta_{n}=\inf _{\mathbf{U} \in \overline{\mathscr{D}}} \frac{\sup _{\overline{\mathbf{v}} \in \overline{\mathscr{D}}} \frac{\overline{\mathbf{V}}^{\mathrm{T}} \overline{\mathbf{G}} \mathbf{U}}{\sqrt{\overline{\mathbf{V}}^{\mathrm{T}} \overline{\mathbf{D}} \overline{\mathbf{V}}}}}{\sup _{\mathbf{V} \in \mathscr{D}} \frac{\mathbf{V}^{\mathrm{T}} \mathbf{G} \mathbf{U}}{\sqrt{\mathbf{V}^{\mathrm{T}} \mathbf{D V}}}}
$$

We show in Appendix III that the following bound for $\theta_{n}(h)$ can be found

$$
\theta_{n}(h) \geqslant \theta_{n}^{*}(h)=\bar{\gamma}_{\min } \sqrt{\frac{\delta_{\min }}{\bar{\delta}_{\max } \gamma_{\max }}}
$$

where $\bar{\gamma}_{\text {min }}$ is the smallest non-zero eigenvalue of $\overline{\mathbf{G}} ; \delta_{\min }$ is the smallest nonzero eigenvalue of $\mathbf{D} ; \bar{\delta}_{\max }$ stands for the largest eigenvalue of $\overline{\mathbf{D}}$; and $\gamma_{\max }$ is calculated as the largest eigenvalue of the matrix $\mathbf{T}=\mathbf{G}^{\mathrm{T}} \mathbf{G}$. Therefore, the following numerical procedure can be applied to study the behaviour of $\theta_{n}$ :

(1) To define the starting space $\Gamma_{h}$, we choose the mesh which was used to obtain the first point, $k=1$, on the graph for the inf-sup test in the $\Gamma_{h}^{\prime}$-norm. Next, we calculate the values $\theta_{n}^{*}, n=1,2,4,8, \ldots, M$ for some $M$, and plot $\lg \left(\theta_{n}^{*}\right)$ vs. $\lg (1 / n)$. 
(2) The sequence described above is repeated for every point, $k=2,3, \ldots$, on the $\Gamma_{h}^{\prime}$-graph, where $M$ may decrease with $k$ (see Section 5), and we check whether the value $\theta_{n}^{*}(h)$ remains bounded from below by some positive constant as $h \rightarrow 0$.

We refer to this scheme as the $\theta_{n}$-test. Note that since $\bar{\gamma}_{\min }$ and $\bar{\delta}_{\max }$ are associated with the starting space $\Gamma_{h}$, the values do not change with $n$, so that they should be calculated only once for every point $k$ (in fact, we can cancel out these terms, rescaling $\theta_{n}$ in (49) by $\sqrt{\bar{\delta}_{\max }} / \bar{\gamma}_{\text {min }}=$ const). Also, we note that $\mathbf{T} \in \mathbb{R}^{\bar{m}} \times \mathbb{R}^{\bar{m}}$; therefore, the only expensive calculation (i.e., solution for the largest eigenvalue of an $m$-by- $m$ matrix) is to get $\delta_{\min }$.

Finally, we note, in summary, that for our complete test to be passed, the inf-sup test in the $\Gamma_{h}^{\prime}$-norm must be passed and the $\theta_{n}$-test must be passed as well.

This numerical test is performed, in the first instance, using uniform meshes, and if not passed, clearly the inf-sup condition is not satisfied. However, in practice, distorted meshes are employed, and therefore the inf-sup test should also be passed in such situations.

While it is important to perform the inf-sup test in the $\Gamma_{h}^{\prime}$-norm for distorted meshes, we deem it sufficient to perform the $\theta_{n}$-test only for uniform meshes.

Hence, for the complete test to be passed, $\beta^{*}(h)$ in (40) must stabilize at a value greater than zero, and $\theta_{n}^{*}(h)$ in (49) must do so too. If this is not the case, strictly, the element under consideration may still be a useful element, but our test was not able to identify it as such. In theory, we then cannot make a definitive judgement regarding the reliability and optimality of the element. However, we can be suspicious that these properties are not present, and indeed our experience tells us that the element will not be reliable.

\section{NUMERICAL EXAMPLES}

\subsection{Problem considered in the tests}

In this section we consider a square plate with all edges clamped, loaded by a uniformly distributed load $p$ (see Figure 2). To compare the results of numerical simulations with our predictions, we assume that the plate is thin enough to obey the Kirchhoff theory. Then, for Poisson's ratio $v=0.3$, the maximal transverse displacement is

$$
w_{\max }=w(L / 2 ; L / 2)=0.00126 \frac{p L^{4}}{D}
$$

The geometry and material characteristics are $L=100 \mathrm{~mm}, E=2 \times 10^{5} \mathrm{MPa}, v=0 \cdot 3, k=5 / 6$.

\subsection{Test predictions}

Following the procedure developed in the previous sections, we apply the tests, given by equations (40) and (49) to meshes in the domain

$$
\Omega=\left\{\left(x_{1}, x_{2}\right) \in(0 ; L)^{2}\right\}
$$

to four- and nine-node displacement-based elements (the QUAD4 and QUAD9 elements) as well as to the elements of the MITC family.

Figure 3 shows the numerical results of the inf-sup test in the $\Gamma_{h}^{\prime}$-norm for uniform meshes, and Figure 4 shows these results for distorted meshes. Figure 5 gives the results of the $\theta_{n}$-test (using 


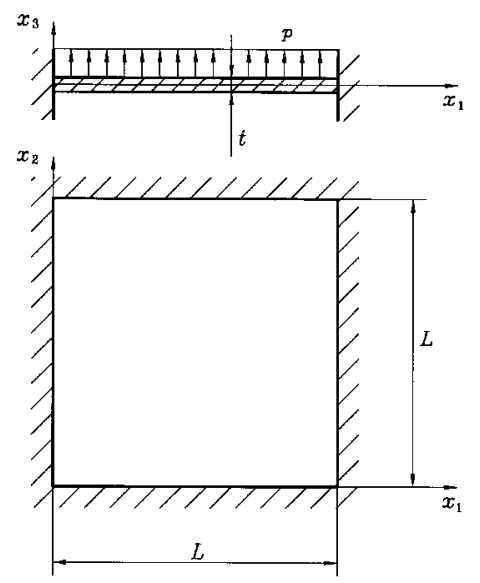

Figure 2. Problem considered in the tests; clamped plate subjected to a uniform load

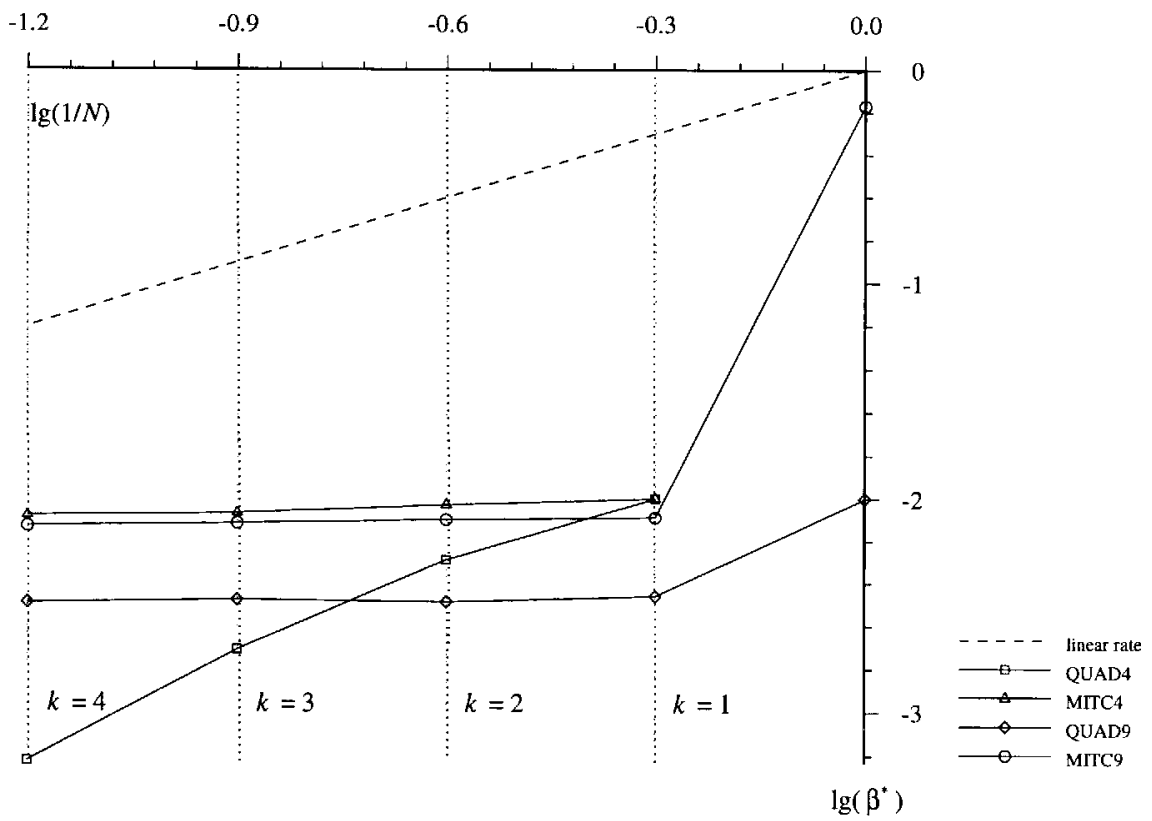

Figure 3. Inf-sup test of quadrilateral plate bending elements in the $\Gamma_{h}^{\prime}$-norm (uniform meshes with square elements). We used $1 \times 1,2 \times 2,4 \times 4,8 \times 8$ and $16 \times 16$ meshes

uniform meshes). The distorted meshes used in all tests (and solutions reported below) are given in Figure 6.

The figures show that the inf-sup test in the $\Gamma_{h}^{\prime}$-norm is not passed for the displacement-based elements when the distorted meshes of Figure 6 are considered. However, the test is passed by 


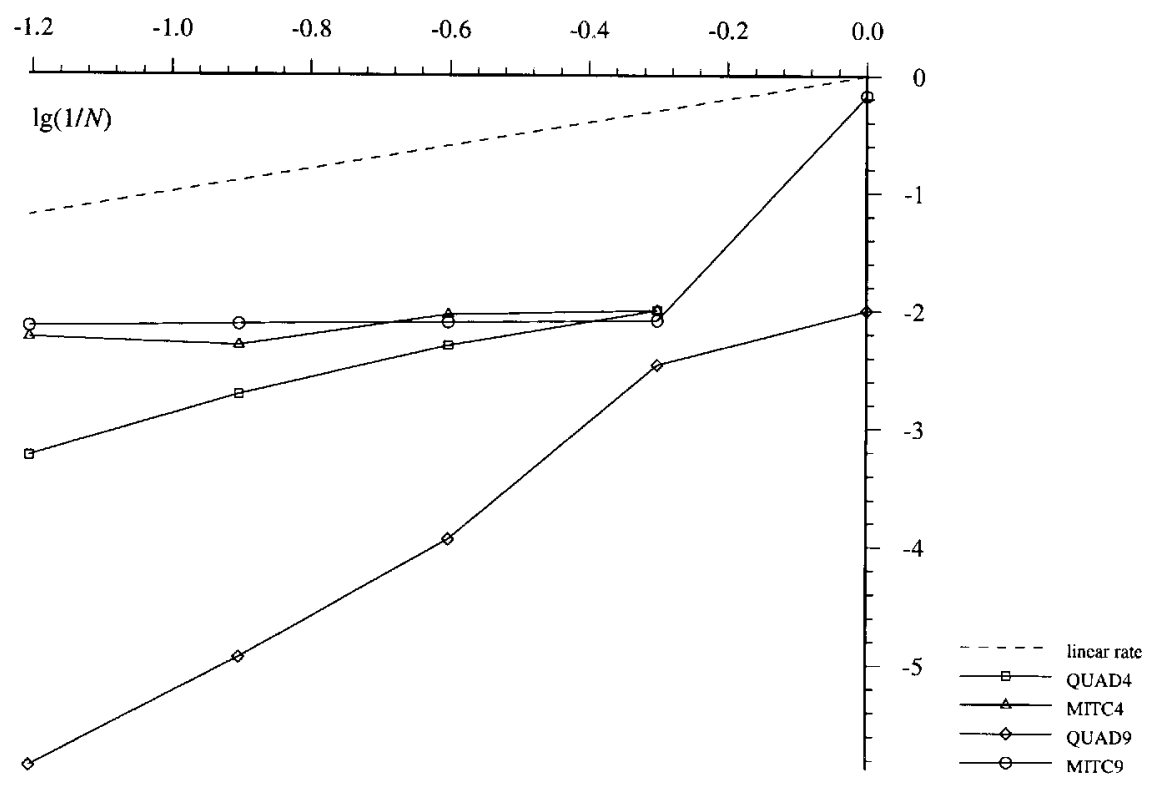

$\lg (\beta)$

Figure 4. Inf-sup test of quadrilateral plate bending elements in the $\Gamma_{h}^{\prime}$-norm (distorted meshes of Figure 6)

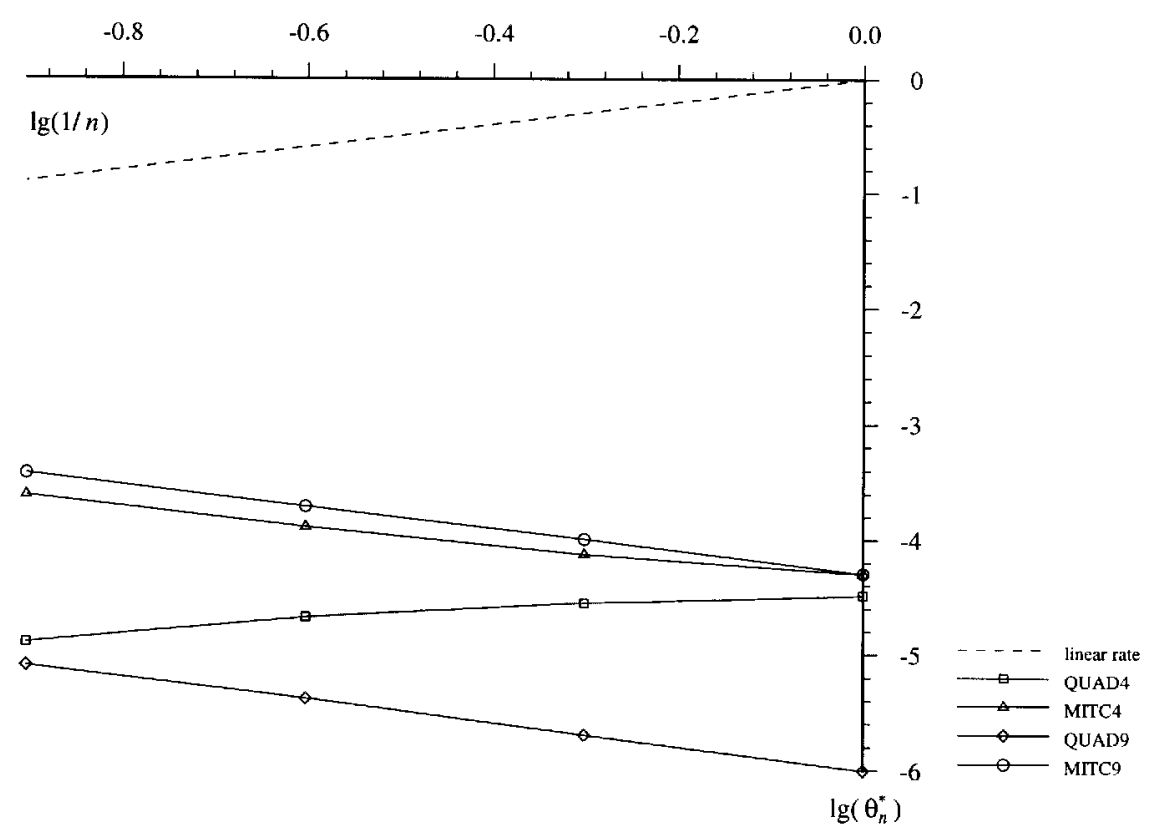

Figure 5. (a) $\theta_{n}$-test of quadrilateral plate bending elements (uniform meshes with square elements). Starting mesh is the $2 \times 2$ mesh $(k=1)$ 


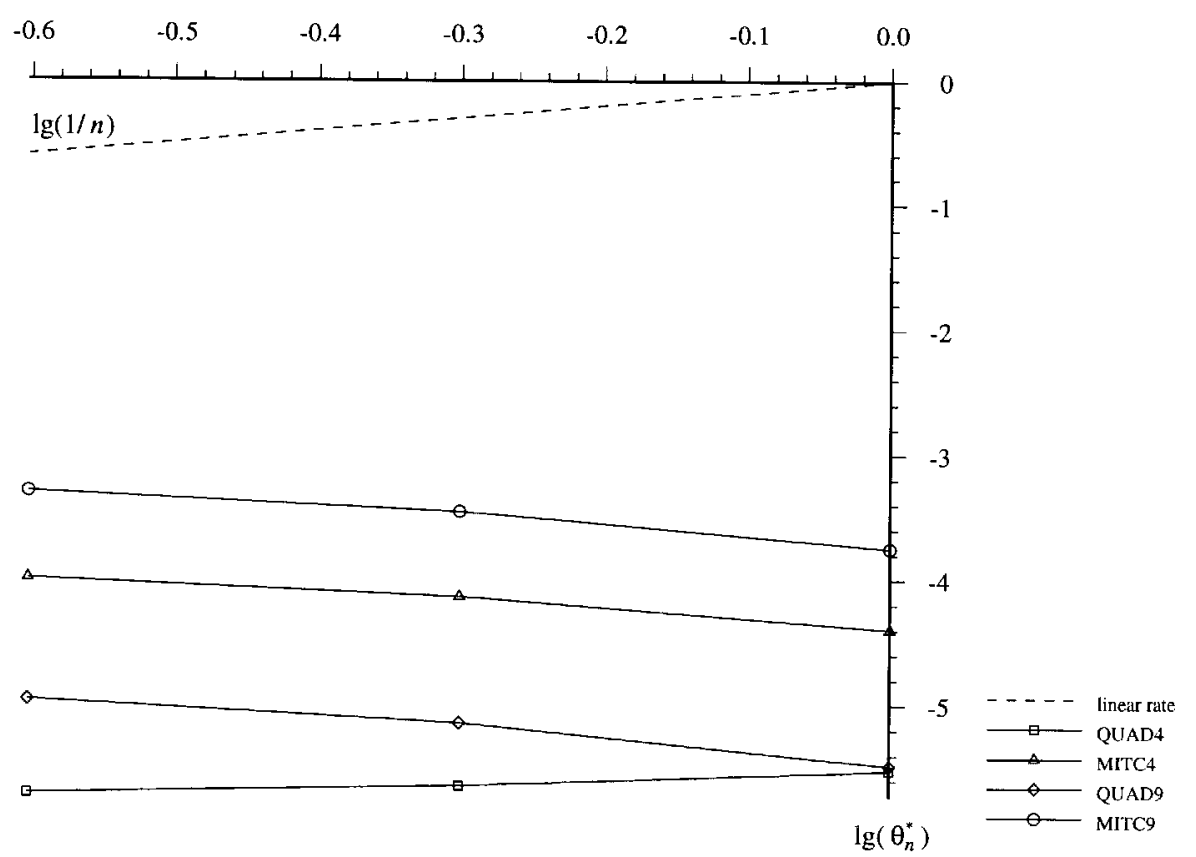

Figure 5. (b) $\theta_{n}$-test of quadrilateral plate bending elements (uniform meshes with square elements). Starting mesh is the $4 \times 4$ mesh $(k=2)$

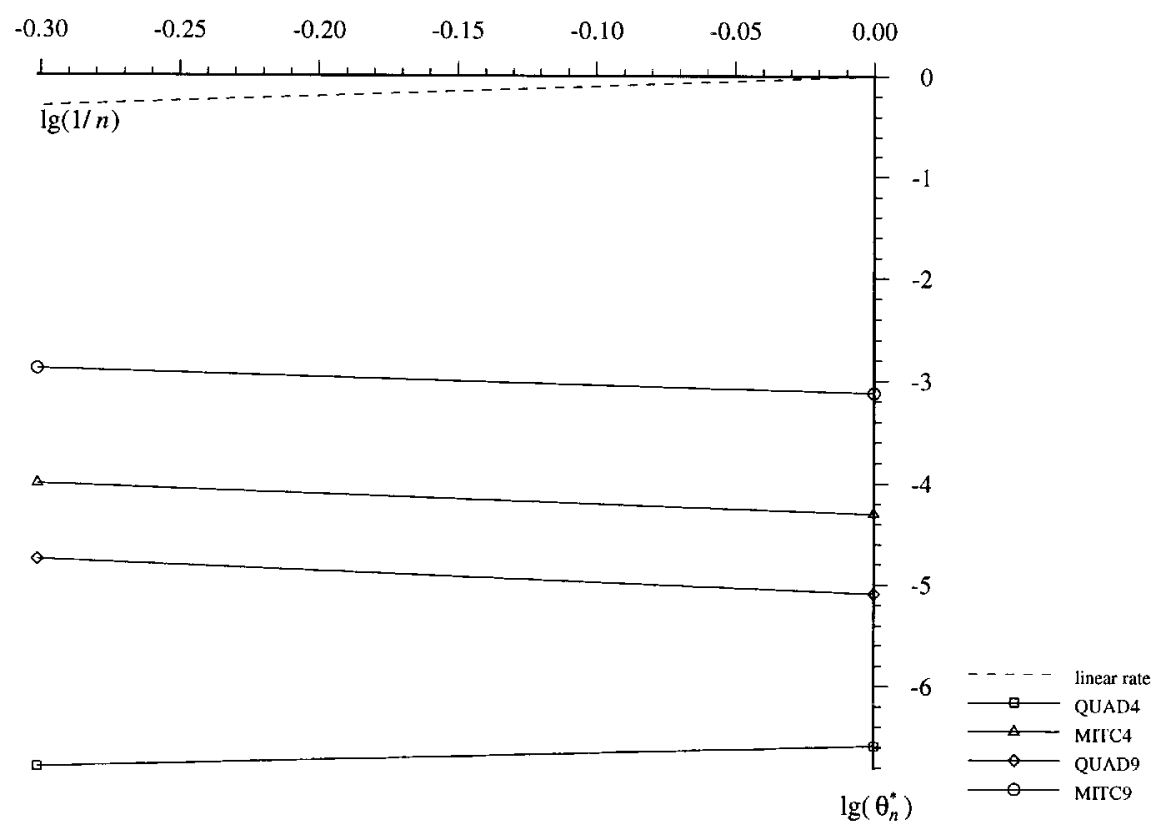

Figure 5. (c) $\theta_{n}$-test of quadrilateral plate bending elements (uniform meshes with square elements). Starting mesh is the $8 \times 8$ mesh $(k=3)$ 


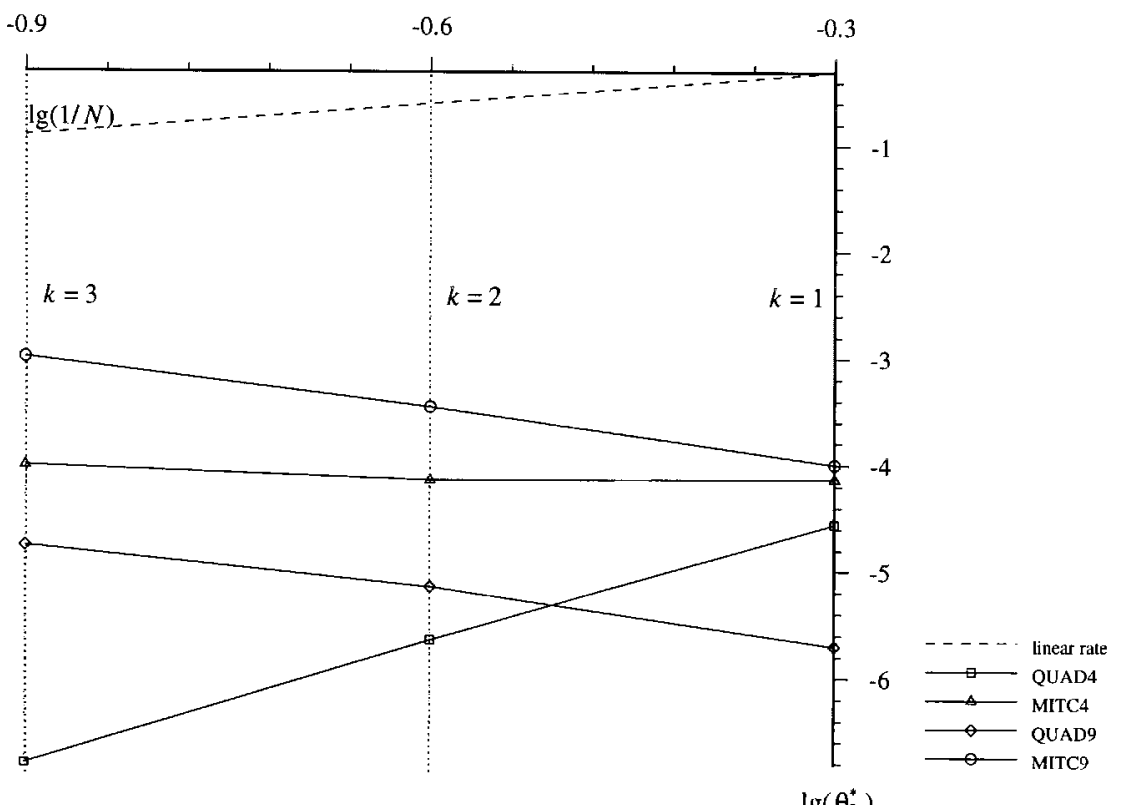

Figure 5. (d) $\theta_{n}$-test of quadrilateral plate bending elements (uniform meshes with square elements). $\theta_{2}^{*}(h)$ values corresponding to $k=1,2,3$

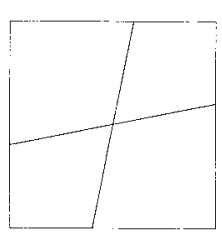

(a)

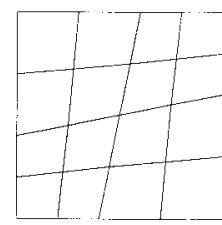

$(c)$

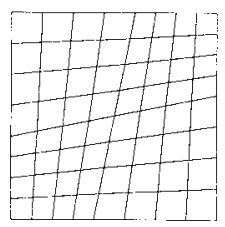

(b)

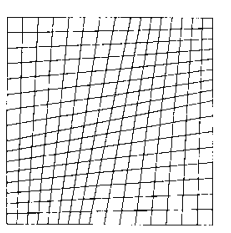

(c)

Figure 6. Distorted meshes used for the tests: (a) $2 \times 2$ mesh; (b) $4 \times 4$ mesh; (c) $8 \times 8$ mesh; and (d) $16 \times 16$ mesh

the nine-node element using uniform meshes. This points out the importance of using distorted meshes in the test.

The $\theta_{n}$-test is also not passed by the four-node displacement-based element. While this test is passed by the nine-node diplacement-based element using uniform meshes, the complete test is not passed because the test in Figure 4 is failed. 

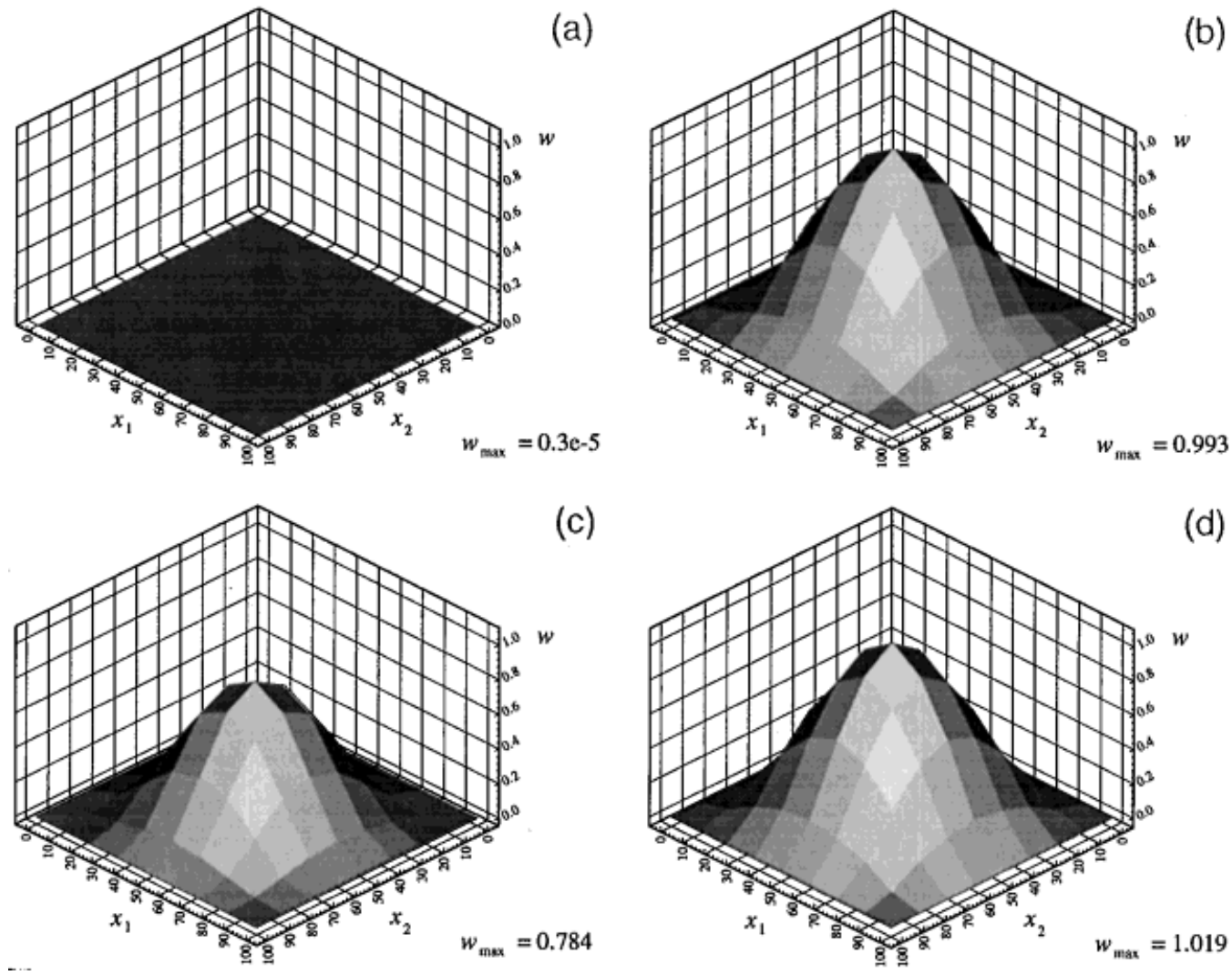

Figure 7. Finite element solution for the transverse displacement $w(\mathrm{~mm})$, for the case $L / t=10,000, p=1 \cdot 454 \times 10^{-7} \mathrm{MPa}$; the Kirchhoff theory solution is $w_{\max }=1 \mathrm{~mm} ; 8 \times 8$ uniform meshes for four-node elements, $4 \times 4$ uniform meshes for ninenode elements: (a) QUAD4 element; (b) MITC4 element; (c) QUAD9 element; (d) MITC9 element

Hence, in summary, we see that the MITC4 and MITC9 elements pass the complete test, while the displacement-based elements fail it.

Figures 7 and 8 show the numerical results for the transverse displacement $w\left(x_{1}, x_{2}\right)$ for the case $L / t=10,000, p=1.454 \times 10^{-7} \mathrm{MPa}$, using uniform and distorted meshes. ${ }^{\dagger}$ There are no surprises in these results, including the observation that the nine-node element locks when the distorted mesh is used. However, here we want to point out that these results are in complete agreement with the results of the inf-sup test, and show once again that the MITC elements work well in contrast to the displacement-based elements.

\footnotetext{
$\dagger$ The solution is depicted using linear interpolation between the mesh nodes, though in fact, the finite element solution for the nine-node elements is quadratic.
} 

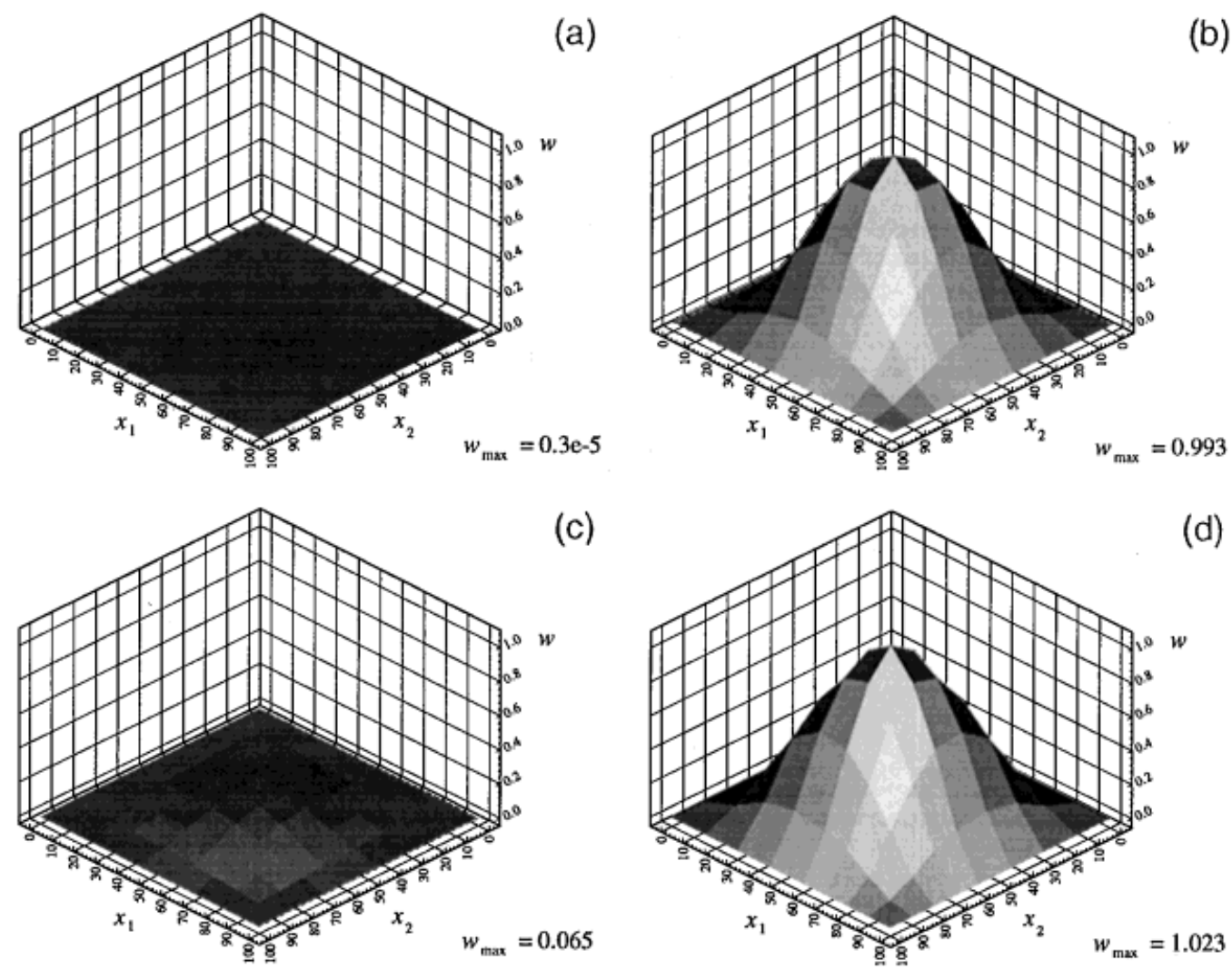

Figure 8. Finite element solution for the transverse displacement $w(\mathrm{~mm})$, for the case $L / t=10,000, p=1.454 \times 10^{-7}$ MPa; the Kirchhoff theory solution is $w_{\max }=1 \mathrm{~mm} ; 8 \times 8$ distorted meshes for four-node elements, $4 \times 4$ distorted meshes for nine-node elements (see Figure 6). (a) QUAD4 element; (b) MITC4 element; (c) QUAD9 element; (d) MITC9 element

\section{CONCLUSIONS}

A test methodology is proposed in this paper, which allows to numerically check whether the inf-sup condition (15) is satisfied for a particular finite element discretization. The developed methodology was applied to a number of elements; the obtained predictions are in agreement with - and go beyond - existing analytical results, and agree with evidence from practical analyses.

Summarizing our findings, we report that:

The nine-node displacement-based element passed the complete test for uniform meshes, but failed the test for a sequence of (reasonably) distorted meshes. This result underlines once more that the element should not be used for the analysis of complex geometries and in general nonlinear analysis. The result also explicitly demonstrates the importance of using distorted elements in the test, in order to identify whether the element under consideration might be effective in general analysis conditions.

The elements of the MITCn family (including the MITC4 element) passed all tests including the test using a sequence of reasonably distorted meshes. While we can expect that the inf-sup 
condition in (15) is, therefore, satisfied for uniform meshes, the effect of distortions should be studied further, because, of course, different kinds of distortions are possible and have different effects. ${ }^{1}$

The theoretical discussion and results presented in the paper show that our test methodology is in general valuable for the evaluation of plate bending elements.

\section{APPENDIX I}

\section{Optimality in the $\Gamma^{\prime}$-norm}

Here we present the important result of optimality of the $\Gamma^{\prime}$-norm for the shear term in (3).

Theorem. There exists $c>0$ independent of plate thickness $t$, such that

$$
\|\gamma(t)\|_{\Gamma^{\prime}} \leqslant c
$$

Proof. If we take $\boldsymbol{\eta}=\boldsymbol{\beta}, \xi=w$, and $\boldsymbol{\varsigma}=\boldsymbol{\gamma}$ in (3), and sum the two equations, we easily have

$$
a(\boldsymbol{\beta}, \boldsymbol{\beta})+\frac{t^{2}}{\mu k}(\gamma, \gamma)=(g, w)
$$

On the other hand, taking $\boldsymbol{\varsigma}=\nabla w$ in the second equation of (3), we have

$$
(\nabla w, \nabla w)=\left(\frac{t^{2}}{\mu k} \gamma, \nabla w\right)+(\boldsymbol{\beta}, \nabla w)
$$

Using twice the inequality $a b \leqslant a^{2}+b^{2} / 4$ (equivalent to $\left.(a-b / 2)^{2} \geqslant 0\right)$ in (52) we have

$$
\|\nabla w\|_{L^{2}}^{2} \leqslant \frac{t^{4}}{\mu^{2} k^{2}}\|\gamma\|_{L^{2}}^{2}+\|\boldsymbol{\beta}\|_{L^{2}}^{2}+\frac{1}{2}\|\nabla w\|_{L^{2}}^{2}
$$

or

$$
\frac{1}{2}\|\nabla w\|_{L^{2}}^{2} \leqslant \frac{t^{4}}{\mu^{2} k^{2}}\|\gamma\|_{L^{2}}^{2}+\|\boldsymbol{\beta}\|_{L^{2}}^{2}
$$

If $t$ is not too big, we have that $\left(t^{4} / \mu^{2} k^{2}\right) \leqslant\left(t^{2} / \mu k\right)$, and (54) gives

$$
\|\nabla w\|_{L^{2}}^{2} \leqslant \frac{2 t^{2}}{\mu k}\|\gamma\|_{L^{2}}^{2}+2\|\boldsymbol{\beta}\|_{L^{2}}^{2}
$$

If we now use (51) and (55) with the Korn inequality

$$
\alpha\|\boldsymbol{\beta}\|_{H^{1}}^{2} \leqslant a(\boldsymbol{\beta}, \boldsymbol{\beta})
$$

the Poincaré inequality

$$
\|w\|_{L^{2}}^{2} \leqslant c_{P}\|\nabla w\|_{L^{2}}^{2}
$$

and duality

$$
(g, w) \leqslant\|g\|_{H^{-1}}\|w\|_{H^{1}}
$$


with $g$ independent of $t$, we can show that

$$
\|\boldsymbol{\beta}\|_{H^{1}}^{2}+t^{2}\|\boldsymbol{\gamma}\|_{L^{2}}^{2}+\|w\|_{H^{1}}^{2} \leqslant c
$$

with $c$ independent of $t$.

Formula (56) is necessary to prove (50). Now we turn to the estimate for $\gamma$. By the Riesz Representation Theorem, we have that for all $\gamma \in \Gamma^{\prime}$ there exists a unique $\chi \in \Gamma$, such that

$$
(\gamma, \chi)=\|\gamma\|_{\Gamma^{\prime}}^{2}=\|\chi\|_{\Gamma}^{2}=\|\gamma\|_{\Gamma^{\prime}}\|\chi\|_{\Gamma}
$$

Our aim is to show that there exists $\boldsymbol{\eta} \in\left[H_{0}^{1}(\Omega)\right]^{2}$ and $\xi \in H_{0}^{1}(\Omega)$ such that

$$
\chi=\boldsymbol{\eta}-\nabla \xi
$$

and

$$
\|\boldsymbol{\eta}\|_{H^{1}}+\|\nabla \xi\|_{L^{2}} \leqslant c\|\chi\|_{\Gamma}
$$

with $c$ independent of $t$. It is clear that (56)-(59) immediately give the desired result: indeed, taking in the first equation of (3) $\boldsymbol{\eta}$ and $\xi$ as in (58), we obtain

$$
\begin{aligned}
(\boldsymbol{\gamma}, \boldsymbol{\chi})=(\boldsymbol{\gamma}, \boldsymbol{\eta}-\nabla \xi) & =a(\boldsymbol{\beta}, \boldsymbol{\eta})-(g, \xi) \\
& \leqslant c\left(\|\boldsymbol{\beta}\|_{H^{1}}+\|g\|_{H^{-1}}\right)\left(\|\boldsymbol{\eta}\|_{H^{1}}+\|\nabla \xi\|_{L^{2}}\right) \leqslant \mathrm{const}\|\boldsymbol{\chi}\|_{\Gamma}
\end{aligned}
$$

and (50) follows using (57).

We are therefore left with the proof of (58) and (59). For this proof, we first consider an auxiliary variational problem of the type (26):

$$
\begin{aligned}
& \text { Find } \boldsymbol{\theta} \in\left[H_{0}^{1}(\Omega)\right]^{2} \text { and } p \in L^{2}(\Omega) \text {, such that } \\
& a^{S}(\boldsymbol{\theta}, \tilde{\boldsymbol{\theta}})+(p, \nabla \cdot \tilde{\boldsymbol{\theta}})=0 \forall \tilde{\boldsymbol{\theta}} \in\left[H_{0}^{1}(\Omega)\right]^{2} \\
& (q, \nabla \cdot \boldsymbol{\theta})=(q, \operatorname{rot} \chi) \forall q \in L^{2}(\Omega)
\end{aligned}
$$

Note that (60) is solvable, since $\chi \in \Gamma$ implies $\chi \cdot \tau=0$ on $\partial \Omega$ and, therefore,

$$
\int_{\Omega} \operatorname{rot} \chi \mathrm{d} \Omega=\int_{\partial \Omega} \chi \cdot \tau \mathrm{d} s=0
$$

In particular, we have

$$
\nabla \cdot \boldsymbol{\theta}=\operatorname{rot} \chi
$$

and

$$
\|\boldsymbol{\theta}\|_{H^{1}} \leqslant c\|\boldsymbol{\chi}\|_{\Gamma}
$$

for some constant $c$ depending only on $\Omega$. 
Now we simply set $\boldsymbol{\eta}=\left[-\theta_{2}, \theta_{1}\right]$; then from (61) and (62) we have

$$
\begin{gathered}
\operatorname{rot} \boldsymbol{\eta}=\frac{\partial \theta_{1}}{\partial x_{1}}+\frac{\partial \theta_{2}}{\partial x_{2}}=\nabla \cdot \boldsymbol{\theta}=\operatorname{rot} \boldsymbol{\chi} \\
\|\boldsymbol{\eta}\|_{H^{1}}=\|\boldsymbol{\theta}\|_{H^{1}} \leqslant c\|\boldsymbol{\chi}\|_{\Gamma}
\end{gathered}
$$

We remark that

$$
\begin{aligned}
& \operatorname{rot}(\boldsymbol{\eta}-\chi)=0 \quad \text { in } \Omega \\
& (\boldsymbol{\eta}-\boldsymbol{\chi}) \cdot \boldsymbol{\tau}=0 \quad \text { on } \partial \Omega
\end{aligned}
$$

which implies the existence of a (unique) $\xi \in H_{0}^{1}(\Omega)$ such that

$$
\nabla \xi=\boldsymbol{\eta}-\chi
$$

From (65) and (53) we immediately have (58) and (59), and the proof is complete.

\section{APPENDIX II}

Derivation of the inf-sup test in the $\Gamma_{h}^{\prime}$-norm

Let us firstly simplify the expression for $\left\|\boldsymbol{S}_{h}\right\|_{\Gamma_{h}^{\prime}}$ as given by equation (38). Since for a positivesemidefinite real-valued $n$-by- $n$ symmetric matrix $\mathbf{A}$ we have

$$
\sqrt{\alpha_{\min }}\|\mathbf{V}\|_{2} \leqslant \sqrt{\mathbf{V}^{\mathrm{T}} \mathbf{A} \mathbf{V}} \leqslant \sqrt{\alpha_{\max }}\|\mathbf{V}\|_{2} \quad \forall \mathbf{V} \in \mathscr{D}=\mathbb{R}^{n} \backslash \operatorname{ker}(\mathbf{A})
$$

where $\alpha_{\min }$ and $\alpha_{\max }$ are the smallest nonzero and the largest eigenvalues of matrix A respectively, we can rewrite (38) as follows:

$$
\left\|\boldsymbol{s}_{h}\right\|_{\Gamma_{h}^{\prime}}=\sup _{\mathbf{W} \in \mathscr{D}} \frac{\mathbf{U}^{\mathrm{T}} \mathbf{G} \mathbf{W}}{\sqrt{\mathbf{W}^{\mathrm{T}} \mathbf{D} \mathbf{W}}} \leqslant \frac{1}{\sqrt{\delta_{\min }}} \sup _{\mathbf{W} \in \mathscr{D}} \frac{\mathbf{U}^{\mathrm{T}} \mathbf{G} \mathbf{W}}{\|\mathbf{W}\|_{2}}=\frac{1}{\sqrt{\delta_{\min }}}\|\mathbf{G} \mathbf{U}\|_{2}
$$

where we made the second transformation invoking the Cauchy-Schwarz inequality for real numbers $^{\ddagger}$ with $\mathbf{W}=\mathbf{U}$.

Substituting (67) into (39), we obtain

$$
\begin{aligned}
\inf _{\mathbf{U} \in \mathscr{D}} \sup _{\mathbf{V} \in \mathscr{D}} \frac{\mathbf{U}^{\mathrm{T}} \mathbf{G} \mathbf{V}}{\sup _{\mathbf{W} \in \mathscr{D}} \frac{\mathbf{U}^{\mathrm{T}} \mathbf{G W}}{\sqrt{\mathbf{W}^{\mathrm{T}} \mathbf{D W}}} \sqrt{\mathbf{V}^{\mathrm{T}} \mathbf{S} \mathbf{V}}} & \geqslant \sqrt{\delta_{\min }} \inf _{\mathbf{U} \in \mathscr{D}} \sup _{\mathbf{V} \in \mathscr{D}} \frac{\mathbf{U}^{\mathrm{T}} \mathbf{G} \mathbf{V}}{\|\mathbf{G} \mathbf{U}\|_{2} \sqrt{\mathbf{V}^{\mathrm{T}} \mathbf{S} \mathbf{V}}} \\
& \geqslant \sqrt{\frac{\delta_{\min }}{\sigma_{\max }}} \inf _{\mathbf{U} \in \mathscr{D}} \sup _{\mathbf{V} \in \mathscr{D}} \frac{\mathbf{U}^{\mathrm{T}} \mathbf{G V}}{\|\mathbf{G} \mathbf{U}\|_{2}\|\mathbf{V}\|_{2}} \\
& =\sqrt{\frac{\delta_{\min }}{\sigma_{\max }}} \inf _{\mathbf{U} \in \mathscr{D}} \frac{\|\mathbf{G} \mathbf{U}\|_{2}}{\|\mathbf{G} \mathbf{U}\|_{2}}=\sqrt{\frac{\delta_{\min }}{\sigma_{\max }}}
\end{aligned}
$$

¥ The Cauchy-Schwarz inequality for two vectors $\mathbf{U}, \mathbf{V} \in \mathbb{R}^{n}$, is

$$
\mathbf{U}^{\mathrm{T}} \mathbf{V} \leqslant\|\mathbf{U}\|_{2}\|\mathbf{V}\|_{2}
$$

and the equality is reached when $\mathbf{U}=\mathbf{V}$

(C) 1997 John Wiley \& Sons, Ltd.

Int. J. Numer. Meth. Engng., 40, 3639-3663 (1997) 
where the first inequality follows from (66); then we used the Cauchy-Schwarz inequality with $\mathbf{V}=\mathbf{U}$, and invoked the condition $\mathbf{U} \in \mathscr{D}$ to cancel out the infimum expression. Summarizing, we obtain

$$
\inf _{\zeta \in \Gamma_{h}} \sup _{\mathbf{v}_{h}=\left(\mathbf{\eta}_{h}, \xi_{h}\right) \in V_{h}} \frac{\left(\boldsymbol{\zeta}_{h}, R_{h}\left(\nabla \xi_{h}-\eta_{h}\right)\right)}{\|\zeta\|_{\Gamma_{h}^{\prime}}\left\|\mathbf{v}_{h}\right\|_{V}} \geqslant \sqrt{\frac{\delta_{\min }}{\sigma_{\max }}}
$$

\section{APPENDIX III}

Derivation of the $\theta_{n}$-test

In order to obtain the infimum in (48), we calculate a lower bound for the expression in the numerator, and an upper bound for the denominator; to do that we firstly apply (66) to the corresponding norm matrices, and secondly, use the Cauchy-Schwarz inequality in the same manner as we did to derive (40):

$$
\begin{aligned}
& \sup _{\overline{\mathbf{v}} \in \mathscr{D}} \frac{\overline{\mathbf{V}}^{\mathrm{T}} \overline{\mathbf{G}} \mathbf{U}}{\sqrt{\overline{\mathbf{V}}^{\mathrm{T}} \overline{\mathbf{D}} \overline{\mathbf{V}}}} \geqslant \frac{1}{\sqrt{\bar{\delta}_{\text {max }}}} \sup _{\overline{\mathbf{V}} \in \overline{\mathscr{D}}} \frac{\overline{\mathbf{V}}^{\mathrm{T}} \overline{\mathbf{G}} \mathbf{U}}{\|\overline{\mathbf{V}}\|_{2}}=\frac{1}{\sqrt{\bar{\delta}_{\max }}}\|\overline{\mathbf{G}} \mathbf{U}\|_{2} \geqslant \frac{\bar{\gamma}_{\min }}{\sqrt{\bar{\delta}_{\max }}}\|\mathbf{U}\|_{2} \\
& \sup _{\mathbf{V} \in \mathscr{D}} \frac{\mathbf{V}^{\mathrm{T}} \mathbf{G} \mathbf{U}}{\sqrt{\mathbf{V}^{\mathrm{T}} \mathbf{D} \mathbf{V}}} \leqslant \frac{1}{\sqrt{\delta_{\min }}} \sup _{\mathbf{V} \in \mathscr{D}} \frac{\mathbf{V}^{\mathrm{T}} \mathbf{G} \mathbf{U}}{\|\mathbf{V}\|_{2}}=\frac{1}{\sqrt{\delta_{\min }}} \sqrt{\mathbf{U}^{\mathrm{T}} \mathbf{G}^{\mathrm{T}} \mathbf{G} \mathbf{U}} \leqslant \sqrt{\frac{\gamma_{\max }}{\delta_{\min }}}\|\mathbf{U}\|_{2}
\end{aligned}
$$

Substituting these expressions into equation (48), and invoking the condition $\mathbf{U} \in \mathscr{D}$, we obtain

$$
\theta_{n}=\inf _{\boldsymbol{s}_{h} \in \Gamma_{h},} \frac{\sup _{\boldsymbol{\chi}_{h} \in \Gamma_{h}} \frac{\left(\boldsymbol{s}_{h}, \boldsymbol{\chi}_{h}\right)}{\left\|\boldsymbol{\chi}_{h}\right\|_{\Gamma_{h}}}}{\sup _{\mathbf{\kappa}_{h} \in \Gamma_{h / n}} \frac{\left(\boldsymbol{s}_{h}, \boldsymbol{\kappa}_{h}\right)}{\left\|\mathbf{\kappa}_{h}\right\|_{\Gamma_{h / n}}}} \geqslant \bar{\gamma}_{\min } \sqrt{\frac{\delta_{\min }}{\bar{\delta}_{\max } \gamma_{\max }}}
$$

\section{ACKNOWLEDGEMENT}

The work of F. Brezzi was partially supported by the HCM Program on Shells, contract ERBCHRXTC 940536.

\section{REFERENCES}

1. K. J. Bathe, Finite Element Procedures, Prentice-Hall, Englewood Cliffs, N.J., 1996.

2. O. C. Zienkiewicz and R. L. Taylor, The Finite Element Method, Vols. 1 and 2, 4th edn, McGraw-Hill, New York, $1989 / 91$.

3. K. J. Bathe and F. Brezzi, 'On the convergence of a four-node plate bending element based on Mindlin/Reissner plate theory and mixed interpolation', in J. R. Whiteman (ed.), The Mathematics of Finite Elements and Applications V, Academic Press Inc., New York, 1985, pp. 491-503.

4. K. J. Bathe and F. Brezzi, 'A simplified analysis of two plate bending elements - the MITC4 and MITC9 elements', Proc. Numerical Methods in Engineering: Theory and Applications, University College, Swansea, UK, 1987.

5. F. Brezzi, K. J. Bathe and M. Fortin, 'Mixed-interpolated elements for Reissner-Mindlin plates', Int. J. Numer. Meth. Engng., 28, 1787-1801 (1989).

6. K. J. Bathe, F. Brezzi and S. W. Cho, 'The MITC7 and MITC9 plate bending elements', Comput. Struct., 32, No. 3/4, 797-814 (1989).

7. K. J. Bathe, M. L. Bucalem and F. Brezzi, 'Displacement and stress convergence of our MITC plate bending elements', Engng. Comput., 7, No. 4, 291-302 (1990). 
8. F. Brezzi, M. Fortin and R. Stenberg, 'Error analysis of mixed-interpolated elements for Reissner-Mindlin plates', Math. Models Meth. Appl. Sci., 1(2), 125-151 (1991).

9. R. Stenberg and M. Suri, 'An $h p$ error analysis of MITC plate elements', SIAM J. Num. Anal., to appear.

10. J. Pitkäranta and M. Suri, 'Design principles and error analysis for reduced-shear plate-bending finite elements', Numer. Math., to appear.

11. F. Brezzi and K. J. Bathe, 'A discourse on the stability conditions for mixed finite element formulations', Comput. Meth. Appl. Mech. Engng., 82, 27-57 (1990).

12. D. Chapelle and K. J. Bathe, 'The inf-sup test', Comput. Struct., 47(4/5), 537-545 (1993).

13. F. Brezzi and M. Fortin, Mixed and Hybrid Finite Element Methods, Springer, Berlin, 1991.

14. B. Häggblad and K. J. Bathe, 'Specifications of boundary conditions for Reissner/Mindlin plate bending finite elements', Int. J. Numer. Meth. Engng., 30, 981-1011 (1990).

15. L. Gastaldi, 'Uniform interior error estimates for the Reissner-Mindlin plate model', Math. Comput., 61, 539-567 (1993).

16. E. N. Dvorkin and K. J. Bathe, 'A continuum mechanics based four-node shell element for general nonlinear analysis', Engng. Comput., 1, 77-88 (1984).

17. K. J. Bathe and E. N. Dvorkin, 'A four-node plate bending element based on Reissner/Mindlin plate theory and a mixed interpolation', Int. J. Numer. Meth. Engng., 21, 367-383 (1985).

18. C. Johnson and J. Pitkäranta, 'Analysis of some mixed finite element methods related to reduced integration', Math. Comput., 38, 375-400 (1982).

19. A. Iosilevich, K. J. Bathe and F. Brezzi, 'Numerical analysis of MITC plate bending elements', Proc. American Mathematical Society Seminar on Plates and Shells, Quebec, Canada, 1996. 